\title{
Thermo-Optical Studies of Laser Ceramics
}

\author{
Oleg V. Palashov ${ }^{1, *}$, Aleksey V. Starobor ${ }^{1}$, Evgeniy A. Perevezentsev ${ }^{1}$, Ilya L. Snetkov ${ }^{1}$ (i), Evgeniy A. Mironov ${ }^{1}$, \\ Alexey I. Yakovlev ${ }^{1}{ }^{(0}$, Stanislav S. Balabanov ${ }^{2}{ }^{-}$, Dmitry A. Permin ${ }^{2}{ }^{-}$and Alexander V. Belyaev ${ }^{2}$ \\ 1 Federal Research Center, Institute of Applied Physics, Russian Academy of Sciences (IAP RAS), 46 Ul'yanov \\ Street, 603950 Nizhny Novgorod, Russia; astarobor@ipfran.ru (A.V.S.); eperevezentsev@gmail.com (E.A.P.); \\ snetkov@appl.sci-nnov.ru (I.L.S.); miea209@rambler.ru (E.A.M.); alexey.yakovlev@ipfran.ru (A.I.Y.) \\ 2 G.G. Devyatykh Institute of Chemistry of High-Purity Substances, Russian Academy of Sciences, 49 Tropinin \\ Street, 603951 Nizhny Novgorod, Russia; balabanov@ihps-nnov.ru (S.S.B.); permin@ihps-nnov.ru (D.A.P.); \\ belyaev@ihps.nnov.ru (A.V.B.) \\ * Correspondence: palashov@appl.sci-nnov.ru; Tel.: +7-(831)-416-0674
}

check for

updates

Citation: Palashov, O.V.; Starobor, A.V.; Perevezentsev, E.A.; Snetkov, I.L.; Mironov, E.A.; Yakovlev, A.I.; Balabanov, S.S.; Permin, D.A.; Belyaev, A.V. Thermo-Optical Studies of Laser Ceramics. Materials 2021, 14 , 3944. https://doi.org/10.3390/ ma14143944

Academic Editor: Heesun Yang

Received: 28 May 2021

Accepted: 13 July 2021

Published: 14 July 2021

Publisher's Note: MDPI stays neutral with regard to jurisdictional claims in published maps and institutional affiliations.

Copyright: (c) 2021 by the authors. Licensee MDPI, Basel, Switzerland. This article is an open access article distributed under the terms and conditions of the Creative Commons Attribution (CC BY) license (https:// creativecommons.org/licenses/by/ $4.0 /)$.
Abstract: A cycle of works on manufacturing and studying laser and magnetooptical ceramics with a focus on their thermo-optical characteristics performed by the research team is analyzed. Original results that have not been published before such as measurements of the Verdet constant in the $\mathrm{Zr}: \mathrm{TAG}, \mathrm{Re}: \mathrm{MgAl}_{2} \mathrm{O}_{4}$, and $\mathrm{ZnAl}_{2} \mathrm{O}_{4}$ ceramics are also presented.

Keywords: thermal effects; laser ceramics; magnetooptical ceramics; ceramics manufacturing

\section{Introduction}

A permanent enhancement of the time-average power of continuous and pulseperiodic lasers leads to a significant increase of the parasitic impact of thermal effects on their operation. The thermal effects arising in laser elements, such as thermally induced depolarization and thermal lens, can result not only in a significant deterioration of the radiation quality or loss increase, but also in a reduced stability of optical devices operation up to a complete destruction of them. The fundamental nature of the problem stimulated the development of various methods for its solution, including the search for alternative optical materials possessing optimal laser, spectral and thermo-optical properties. One of the trends nowadays is the creation of laser ceramics.

The principal advantages of ceramics are the possibility of creating large-aperture (like in glass) elements, maintaining of a high (like in a single crystal) thermal conductivity, and the potential of creating ceramics from media that cannot be grown at the present in the form of a single crystal but have unique end user properties facilitated the development of the adequate technologies. Modern technologies enable production of laser elements from ceramics with a sufficient optical quality, a large aperture and a high concentration of dopants. However, this new type of laser materials has features which have no analogues either in glasses or in single crystals and which can negatively manifest themselves in propagation of high time-average power laser radiation through ceramics. Since in a deformed single crystal, the quality of the transmitted radiation depends on the orientation of the crystallographic axes, in ceramics (the grains of which have an arbitrary orientation), the distortion pattern is averaged on grains on ray path. Even cubic crystal ceramics, that may seem to be indistinguishable from similar single crystals, in the presence of mechanical stress (caused, for example, by heating), acquires phase distortions, the inhomogeneity scale of which is comparable to the grain size $l_{\mathrm{g}}$. This occurs because the rays located at a distance $\sim l_{\mathrm{g}}$ from each other pass through their statistically independent set of grains. These distortions, in turn, lead to the dispersion of thermal depolarization, which degrades the polarization quality of the radiation and the dispersion of thermally induced phase distortions (thermal lens). Thus, both the polarized and the depolarized parts of the 
radiation that has passed through the thermally loaded ceramic element will have a smallscale modulation with a characteristic size of $\sim l_{\mathrm{g}}$ and an amplitude dependent on the ratio of the sample length to the longitudinal grain size. The theory of thermally induced birefringence in ceramics was constructed and developed in detail in [1-3] (both in initially isotropic elements [1,3] and in gyrotropic elements [2]) and was confirmed experimentally in [4-6]. In this paper we present results in which the dispersion of thermally induced depolarization and the dispersion of thermally induced phase distortions are negligible.

The use of the ceramics in high-power lasers is currently regarded by the scientific community to be highly promising and the study of the thermal effects in ceramic laser elements is quite relevant. Thermal effects play a significant role both in the generation and amplification of high-power laser radiation in active elements and in the propagation of such radiation through passive optical elements. The calculation, prediction, and selection of methods for reducing and compensating thermal effects is an indispensable part of the development of such laser systems. Therefore, for assessing the potential and specific features of application of new optical materials, it is necessary to determine their material constants.

The absorption of radiation in the optical element bulk leads to an inhomogeneous temperature distribution. As a result, all temperature-dependent optical characteristics (refractive index, thermal conductivity coefficient, Verdet constant, etc.) become inhomogeneously distributed and the internal stresses and, hence, the deformations will lead to the appearance of thermally induced birefringence caused by the photoelastic effect. The inhomogeneous distribution of the refractive index together with the change in geometric dimensions caused by the deformation of the optical elements lead to wave front distortion referred to as the "thermal lens". The aberrations induced by the thermal lens do not lead to polarizing distortions of the laser radiation, but affect the mode composition of the radiation passing through the optical element [7]. The optical power of the thermal lens D is determined by the ratio [7]:

$$
D=\frac{\alpha L P}{4 \pi \kappa a^{2}} P_{\text {las }}
$$

where $\alpha$ and $\kappa$ are the coefficients of the medium absorption and thermal conductivity, $P$ is the thermo-optical constant [8], $\mathrm{L}$ is the length of the optical element, $\lambda$ is the wavelength. Plas is the power of incident radiation, and $a$ is the laser beam radius.

The depolarization $\gamma_{P}$ in a material with a cubic crystal lattice depends on the crystallographic axes orientation relative to the polarization of the incident radiation which is related to the anisotropy of the stress-optic tensor. The transverse structure of the thermal depolarization $\gamma_{P}$ with a fourth-order symmetry axis has the shape of the so-called "clover leaf", more often called the "Maltese cross". For MAE made of cubic crystal in [001] orientation (in presence of Faraday effect, and angle of rotation of polarization plane $\varphi=45^{\circ}$ ), minimal value of $\gamma_{P}$ can be calculated by the formula [9]:

$$
\gamma_{P}=A_{p} p^{2} \min \left(1, \xi^{2}\right),
$$

where $A_{p}$ is the coefficient dependent on the laser beam profile $\left(A_{p}=0.0139\right.$ for a Gaussian beam and $A_{p}=0.0085$ for a flat-top beam and $p$ is the normalized laser power:

$$
p=\frac{\alpha Q L P_{\text {las }}}{\lambda \kappa}
$$

where $Q$ is the thermo-optical constant responsible for depolarization and $\xi$ is the optical anisotropy parameter $\xi\left(\xi=\pi_{44} /\left(\pi_{11}-\pi_{12)}\right.\right.$, where $\pi_{i j}$ are elements of stress-optic tensor in Nye notation; for amorphous media $\xi=1$ ). The transition to other crystallographic orientations is reduced to the following replacements: $\xi \rightarrow 1$, in the case of [111] orientation, $Q \rightarrow Q(1+2 \xi) / 3[9,10]$, and for the ceramic element $Q=Q(2+3 \xi) / 5$ [11] or $Q=Q(53+75 \xi) / 128$ [2], depending on the grain statistics. For the ceramics, one more term inversely proportional to the number of grains on the laser beam path and to $p^{2}$ is 
added to the expression (2). However, as the characteristic size of the grain in the studied ceramics was small compared to the sample length, this term was neglected.

Thus, the material constants responsible for thermal effects, such as the optical anisotropy parameter $\xi$ and the thermo-optical constants $P$ and $Q$ play an important role in the calculation of thermal effects in lasers with a high average power [8]. For crystals, maximum information about material constants can be obtained from the analysis of thermal effects in the [001] orientation. In this case, the parameter $\xi$ and a combination of the material constants $\alpha_{0}|Q| / \kappa$ and $\alpha_{0} P / \kappa$ may be measured separately. If the values of the thermal conductivity $\mathrm{K}$ and the linear absorption coefficient $\alpha$ are known, the values of the thermo-optical characteristics $|Q|$ and $P$ of the material under study may be determined independently. The value of $P$ can be found from Equation (1) using the value of the thermal lens optical power measured in experiment, and the value of $|Q|$ from measurements of the minimal thermal depolarization $\gamma$. The situation is different for ceramic materials. In this case, only a more complex combination $Q_{e f f}=Q X$ can be found from the dependence of thermally induced depolarization on laser power, whereas the thermo-optical characteristics cannot be differentiated. However, this information may be sufficient for comparison of the new material with the widely used ones to date.

The team of the authors has been actively involved in the development and investigation of various laser ceramics for many years. A specific feature of our work is the theoretical and experimental study of the thermo-optical properties of ceramics, which, in our opinion, are of key significance for lasers with a high time-average power. That is why the title of this article is "Thermo-optical studies of laser ceramics", although we also consider other properties of ceramics (magneto-optical, spectral, laser) and do not claim that our research is comprehensive.

Two kinds of laser elements: active elements (AE) of laser heads/amplifiers and magneto-active elements (MAE) of optical isolators (Faraday isolators (FI)) are most strongly subject to thermal self-action due to the inevitable and relatively high absorption of radiation. Two main chapters of the represented work are devoted to them.

\section{Magneto-Active Ceramics}

Faraday isolators are the key elements of laser schemes that are aimed to protect their components from inevitable back reflection that is very dangerous in the work with high-power laser radiation. The main part of the FI is a MAE placed in the magnetic field $H$ produced by a permanent magnet system. The Faraday effect leads to the circular birefringence, appearance in the MAE as a result of which the plane of polarization of laser radiation is rotated by an angle $\varphi$ defined by

$$
\varphi=V \int_{0}^{L} H d z,
$$

where $V$ is the Verdet constant. For proper FI operation the angle $\varphi$ must be equal to $45^{\circ}$ and, in an ideal case, the beam is fully reflected by the polarizer on the reverse passage.

Thus, the Verdet constant is one of the most important characteristics of the magnetoactive medium as it specifies its length needed to attain the angle $\varphi=45^{\circ}\left(L_{45}\right)$. This length, in turn, determines thermally induced distortions of the radiation. For paramagnetic media, the Verdet constant can be increased by cooling the MAE. We studied the spectral and temperature dependences of the Verdet constant for magneto-active ceramics, the obtained results are presented in the work.

Another important FI characteristic is the isolation ratio that is determined by polarization distortions. The isolation ratio $I$ is measured in decibels and is defined as

$$
I[d B]=-10 \lg (\gamma),
$$


where the radiation depolarization $\gamma$ is understood as:

$$
\gamma=P_{d} / P_{\text {las }}
$$

where $P_{d}$ is the power of the radiation component polarized orthogonally to the incident radiation by the power $P_{\text {las }}$. The "cold" depolarization arising in MAEs due to the inhomogeneous and imperfect optical element is small as a rule $\left(10^{-5}-10^{-4}\right)$. The radiation depolarization caused by absorption in optical elements is called "hot" or "thermally induced" depolarization. With increasing laser radiation power, the depolarization increases too and at a certain power it reaches a threshold value for practical application (typically 0.001 , or $I=30 \mathrm{~dB}$ ), usually referred to as maximum permissible power $P_{\max }$.

The inhomogeneous over the radius distribution of the angle of rotation of the plane of polarization caused by the temperature dependence of the Verdet constant leads to a change in the path difference between two circular eigenpolarizations without changing them. The degree of depolarization determined by the dependence of the Verdet constant on the transverse coordinates $\left(\gamma_{V}\right)$ can be calculated by the expression [12]:

$$
\gamma_{V}=B\left(\frac{\varphi}{\pi / 4} \frac{\alpha P_{l a s}}{\kappa} \frac{1}{T}\right)^{2},
$$

Here, $B$ is the numerical coefficient dependent on the laser beam shape that is $B=0.00104$ for a Gaussian beam, it is assumed that Verdet constant $V$ is inversely proportional to temperature $T:\left|V / \frac{d V}{d T}\right|=T$.

The thermally induced birefringence induced by the photoelastic effect changes the path difference between the eigenpolarizations and the eigenpolarizations themselves that become elliptical at each point. Both effects lead to an inhomogeneous change of the plane of radiation polarization and depolarization.

The contribution of the photoelastic effect to thermally induced depolarization $\gamma_{P}$ is much higher, and the depolarization $\gamma_{V}$ due to dependence of the Verdet constant on the transverse coordinates may be neglected [13]. However, in some cases (e.g., in a cryogenic FI) the contribution of the photoelastic effect may be several times weaker. In the work [14] we defined the element length $L^{*}$ at which the magnitudes of depolarizations of both effects become equal:

$$
L^{*}=\pi \sqrt{\frac{B}{A}}\left|\frac{\lambda}{Q V} \frac{d V}{d T}\right| .
$$

For example, for an MAE made of terbium-gallium garnet (TGG) at $\mathrm{T}=77 \mathrm{~K}$, $L^{*} \approx 5 \mathrm{~mm}$, while the length of the crystal used in a cryogenic Faraday isolator is $L \approx 3 \mathrm{~mm}$. Therefore, $\gamma_{V}$ must be taken into account when estimating thermally induced depolarization, which was confirmed in the work [15], where laser radiation depolarization $\gamma_{V}$ caused by the transverse inhomogeneity of the Verdet constant at MAE heating was revealed and measured for the first time.

If a paramagnetic (for example, TGG) is used as MAEs' material, the isolation ratio of the device may additionally reduce under the parasitic influence of the effect of paramagnetic MAE magnetization that becomes significant when the magnetic field inhomogeneity inside the element due to magnetization is comparable with the inhomogeneity of the magnetic system field (we showed this effect in the work [16]). Such a situation usually occurs in large-aperture and cryogenic FIs. A new type of a Faraday isolator in which the azimuthally symmetric component of polarization distortions (both under the influence of the effect of paramagnetic MAE magnetization and caused by the transverse inhomogeneity of the Verdet constant at MAE heating) can be made significantly weaker by creating magnetic field inhomogeneity was proposed and demonstrated experimentally in Ref. [12].

We have considered above the features of thermo-optical distortions in an FI. Further, we will analyze the results of our studies of a number of magneto-optical ceramics. We will 
take as a reference a TGG crystal as it is currently the most widely used magneto-optical material.

\subsection{TGG Ceramics}

TGG ceramics is based on terbium gallium garnet $\left(\mathrm{Tb}_{3} \mathrm{Ga}_{5} \mathrm{O}_{12}\right)$. The physical properties of the TGG crystal and TGG ceramics are largely the same: the Verdet constant [17], thermal conductivity, thermo-optical ratio $\mathrm{d} n / \mathrm{d} T$, and thermal expansion coefficient [18-20]. We studied experimentally thermally induced distortions in samples of TGG ceramics produced by Konoshima, Japan [20-23]. We measured the power and temperature dependence of the isolation ratio and optical power of the thermal lens $D$ in the $300 \ldots 90 \mathrm{~K}$ range. Also, we developed FIs with different optical schemes, including schemes with compensation of thermally induced depolarization.

The dependence of thermally induced depolarization in TGG ceramics on radiation power in a $9.1 \mathrm{~mm}$-long sample and in a $5 \mathrm{~mm}$-long sample with increased absorption is shown in Figure $1[20,23]$. The diameter of all samples was $10 \mathrm{~mm}$. Graphs for TGG crystals with [001] orientation (circles and a solid curve) [24] and with [111] orientation (dashed curve) is given for comparison. Assuming that the thermo-optical characteristics of TGG ceramics are like in the single crystal, we calculated the radiation absorption coefficient to be $\alpha=0.0014 \mathrm{~cm}^{-1}$, which corresponds to the absorption coefficients of commercial samples of TGG crystals. The thermal lens induced in the samples also corresponds to the value for the TGG single crystal [20]. The dependence of the optical power of the thermal lens $D$ on the radiation power from [25] for TGG ceramics, re-calculated for the length $L=L_{45}$ in a magnetic field of $H=2.5 \mathrm{~T}$ and a beam having an equal diameter (of $2.4 \mathrm{~mm}$ ) is plotted in Figure 2. Within the 88-300 K temperature range, the thermally induced depolarization in magnetic field of $1.9 \mathrm{~T}$ in a sample of TGG ceramics reduces by more than 6 times on cooling, and $D$ by more than 2 times [23].

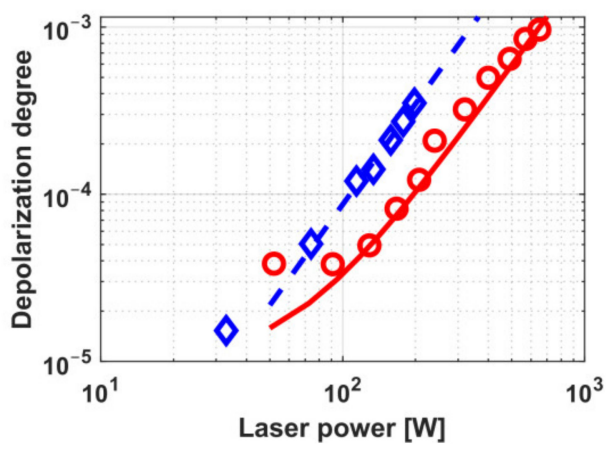

(a)

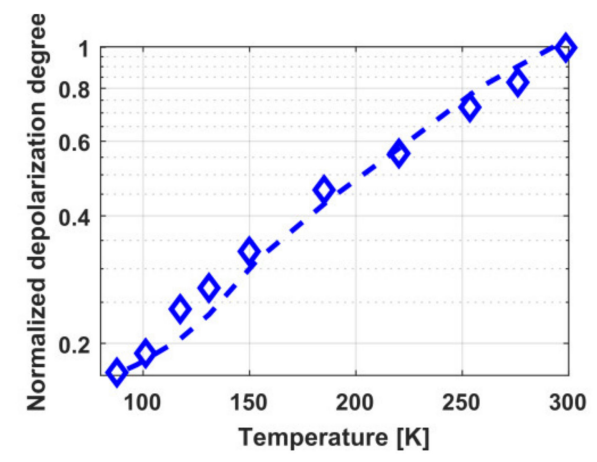

(b)

Figure 1. Thermally induced depolarization in TGG ceramics (diamonds-experimental points, dashed curve-theoretical approximation) as a function of laser radiation power at room temperature (a) and temperature (b). The graph for TGG crystals (circles-experimental points, solid curvetheoretical approximation) with [001] orientation from [24] is given for comparison.

With the use of a TGG ceramics having length $9 \mathrm{~mm}$ as a MAE of the FI with a magnetic system with a maximal magnetic field strength of $2.5 \mathrm{~T}$, the maximal power $P_{\max }$ at which the isolation ratio of $30 \mathrm{~dB}$ may be attained is $340 \mathrm{~W}$ (Figure 1a) [20], which is about twice inferior to that for the TGG crystal with [001] orientation equal to $600 \mathrm{~W}$ [24]. Thermally induced depolarization depends on crystal orientation and the optical anisotropy parameter $\xi$. For the case when $\xi>0$, the optimal orientation is [001] [9], but in view of random grain orientation in ceramics, the properties of the medium are close to the crystal with [111] orientation and it introduces significant depolarization. 


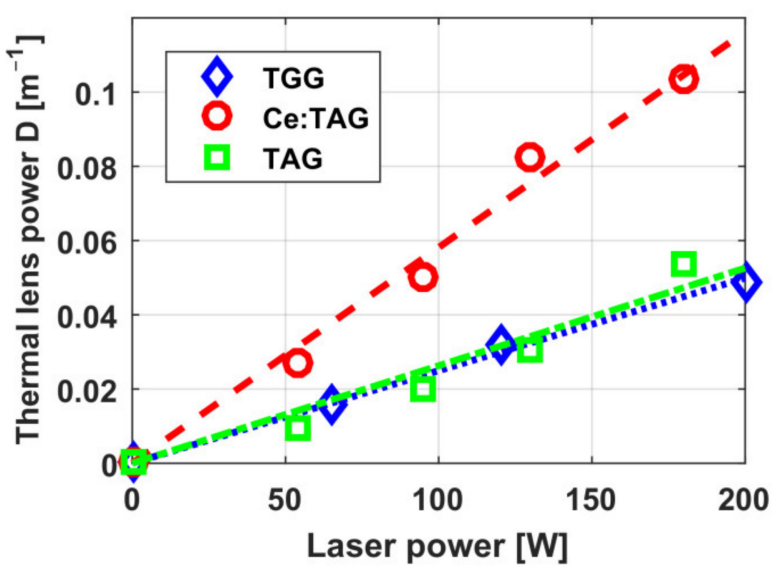

Figure 2. The optical power of thermal lens $\mathrm{D}$ as a function of laser radiation power in TGG and TAG-based FIs, Ce(0.1\%):TAG.

A model cryogenic Faraday isolator was also constructed on TGG ceramics with increased absorption $(\alpha=0.00561 / \mathrm{cm})$ and a length of $5 \mathrm{~mm}$, ensuring an isolation ratio of $28 \mathrm{~dB}$ at a power of $450 \mathrm{~W}$. In this case, there arises thermal lens having optical power $D=0.6 \mathrm{~m}^{-1}$ [23]. Taking into consideration that the optical element of the FI is shortened due to increasing Verdet constant on cooling, the gain in $\gamma$ is a factor of 30, and in $D$ a factor of 5. Parameter optimization (reduction of MAE length to 3.5-4 mm and the use of samples with $1.4 \cdot 10^{-3} \mathrm{~cm}^{-1}$ absorption) of a ceramic sample will enable a $P_{\max }$ increase up to $\sim 1.8 \mathrm{~kW}$.

The schemes with compensation of thermally induced depolarization in a Faraday isolator using ceramic MAEs were first implemented on TGG ceramics in the works [21,22]. Two schemes were considered: with internal [26] and external [27] compensation. The idea underlying these schemes implies the use of two optical elements, in which there arises thermally induced depolarization, and a polarization rotator between them that reverses the sign of the birefringence, thus partially compensating the thermally induced depolarization. In the scheme with internal compensation, the MAE is divided into two identical elements between which a reciprocal polarization rotator is placed. In the case of external compensation, besides a conventional $\mathrm{MAE}$, a reciprocal rotator and an additional optical element (AOE) that does not rotate the polarization plane are used. The principal advantage of this scheme is a possibility to modify traditional FIs avoiding changes in their magnetic system and MAE and to use as an $\mathrm{AOE}$ a different, not necessarily magneto-active material [27]. Internal compensation permitted reaching the isolation ratio up to $35 \mathrm{~dB}$ at a radiation power of $740 \mathrm{~W}$ [22]. External compensation enabled an increase in the isolation ratio at a power of $340 \mathrm{~W}$ from 30 to $38 \mathrm{~dB}$ [21]. According to the estimates, $P_{\max }>2 \mathrm{~kW}$ in both schemes using optimized MAE length. The dependence of the depolarization degree on the radiation power for both compensation variants is presented in Figure 3.

Thus, TGG ceramics is comparable to TGG crystal as a medium for FIs for lasers with high average power, including the cases of nitrogen temperatures. At the same time, it allows producing large-aperture Faraday isolators operating with high-power laser radiation, which opens up new opportunities for creating lasers with high time-average and peak power. 


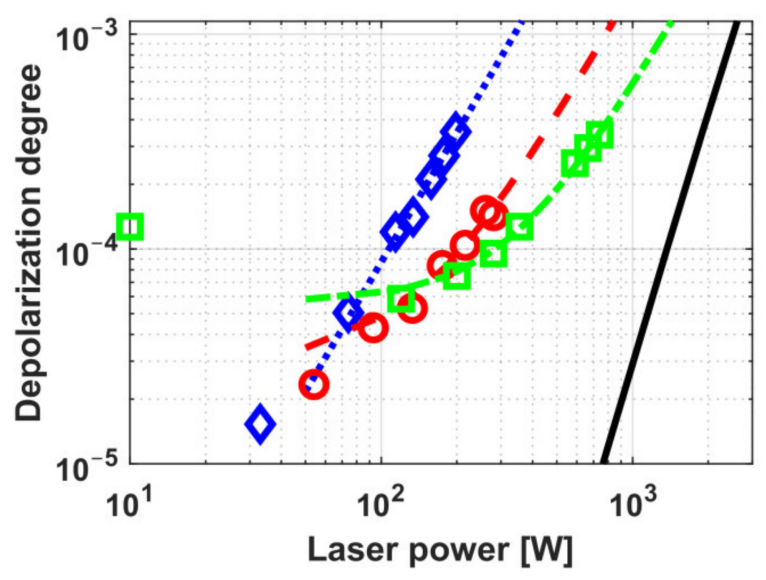

Figure 3. Thermally induced depolarization as a function of laser radiation power in FI based on TGG ceramics without compensation (diamonds-experimental points, dotted curve-theoretical approximation) and with internal (squares and dash-dotted curve) and external (circles and dashed curve) compensation. The solid curve is the theoretical dependence for internal compensation for optical MAE parameters.

\subsection{Re:TAG Ceramics}

One of the materials promising for use in FIs is terbium aluminium garnet $\mathrm{Tb}_{3} \mathrm{Al}_{5} \mathrm{O}_{12}$ (TAG) that surpasses TGG in the value of the Verdet constant by 37\% [28-30]. However, it is very difficult to grow TAG single crystals with acceptable aperture because of their incongruent melting nature and unstable TAG phase in the $\mathrm{Tb}_{2} \mathrm{O}_{3}-\mathrm{Al}_{2} \mathrm{O}_{3}$ system [31]. Several research teams have been developing various methods for growing TAG crystals, but their optical aperture still does not exceed several millimeters [30,32]. Therefore, we believe that an optimal way to obtain such a medium is to produce TAG ceramics. The first results in manufacturing TAG ceramics samples, as well as the study of the optical quality, microstructure, magneto-optical property, and thermal conductivity were reported in [33]. The technology of ceramics allows producing optical elements made of TAG ceramics of arbitrary size, up to tens of centimeters in cubic garnet phase, avoiding problems related to crystal phase instability in melt [34]. Currently, several teams manufacture high-quality TAG ceramics employing different methods [35-37]. In the experiments described in this section, the ceramics produced by the Chinese institutes SIOM (Shanghai, China) [37] and SICCAS (Shanghai, China) [35] was used.

The technology of growing ceramics allows introducing into its composition both sintering additives, that increase the transparency of the medium, and additional ions, that can affect the Verdet constant. We investigated TAG ceramic samples doped with Ce, Pr, Ho, $\mathrm{Si}, \mathrm{Ti}$, and $\mathrm{Zr}$ elements with a concentration of up to $2 \mathrm{at} \%$. According to the measurements, the value of the Verdet constant in the studied ceramic samples does not depend on the dopant type and concentration to an accuracy of the experimental error. However, in the literature there is a wide scatter in the values of the measured Verdet constant both in pure and doped TAG, which in some cases leads to contradictory conclusions about the dependence or independence of the Verdet constant on dopants. This is especially true if the effect is rather week $(\sim 10-15 \%)$ or the doping is low (1-5 at.\%). The literature sources and possible causes of the significant scatter in the Verdet constant values were analyzed in the work [38], including

- the temperature dependence of $\mathrm{V}(\sim 1 / \mathrm{T})$ in paramagnetic materials, to which TAG belongs;

- the accuracy of determining the wavelength of radiation sources;

- the accuracy of determining the angle of rotation of the polarization plane;

- the accuracy of determining the magnitude/integral of the magnetic field and the position of the sample in it; 
- a high unaccounted concentration of sintering additives and impurities that may exceed the concentration (or influence) of the declared dopants, etc.

The spectral dependence $V(\lambda)$ of TAG ceramics may be approximated for all samples to an accuracy better than $5 \%$ in the form:

$$
V(\lambda)=\frac{C}{\lambda^{2}-\lambda_{0}^{2}}
$$

where the values of the coefficients $C$ and $\lambda_{0}$ for the studied ceramics are $C=5.33 \times 10^{7}\left(\mathrm{rad} \times \mathrm{nm}^{2}\right) /(\mathrm{T} \times \mathrm{m})$ and $\lambda_{0}=290 \mathrm{~nm}$. The experimental values for the Verdet constant at the wavelengths 633, 808 and $1064 \mathrm{~nm}$ are given in Figure 4. The spectral dependence of the Verdet constant in TAG compared to TGG ceramics and other media is presented in Figure 5.

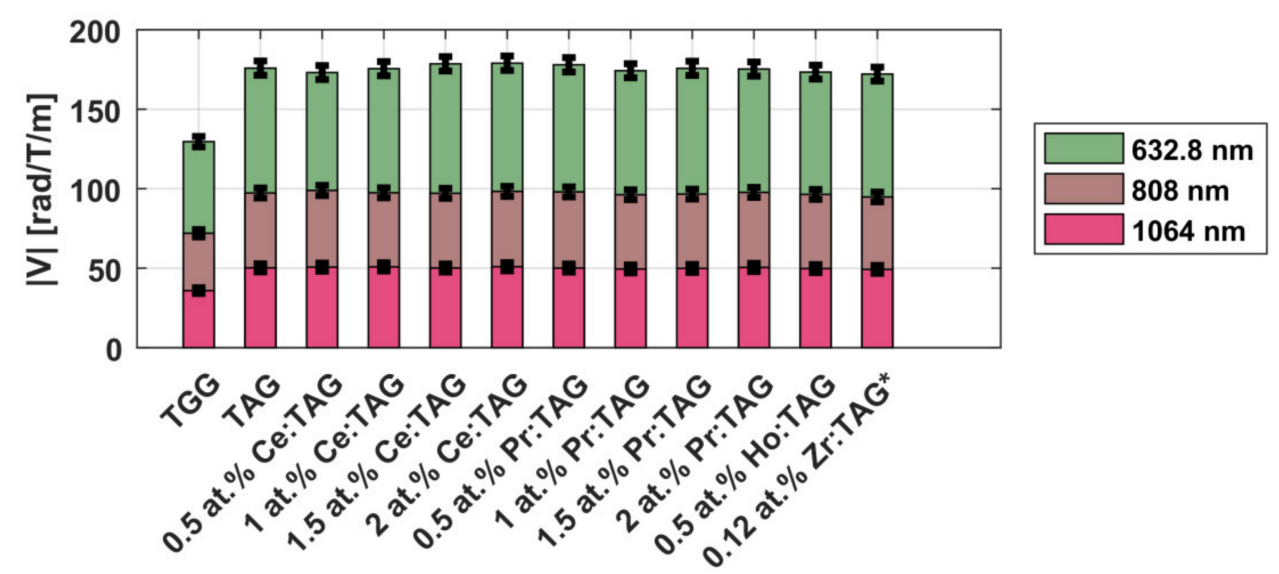

Figure 4. Verdet constant of the TGG and Re:TAG ceramics samples at the wavelengths 1064, 808 and $633 \mathrm{~nm}$ [38]. * - unpublished results.

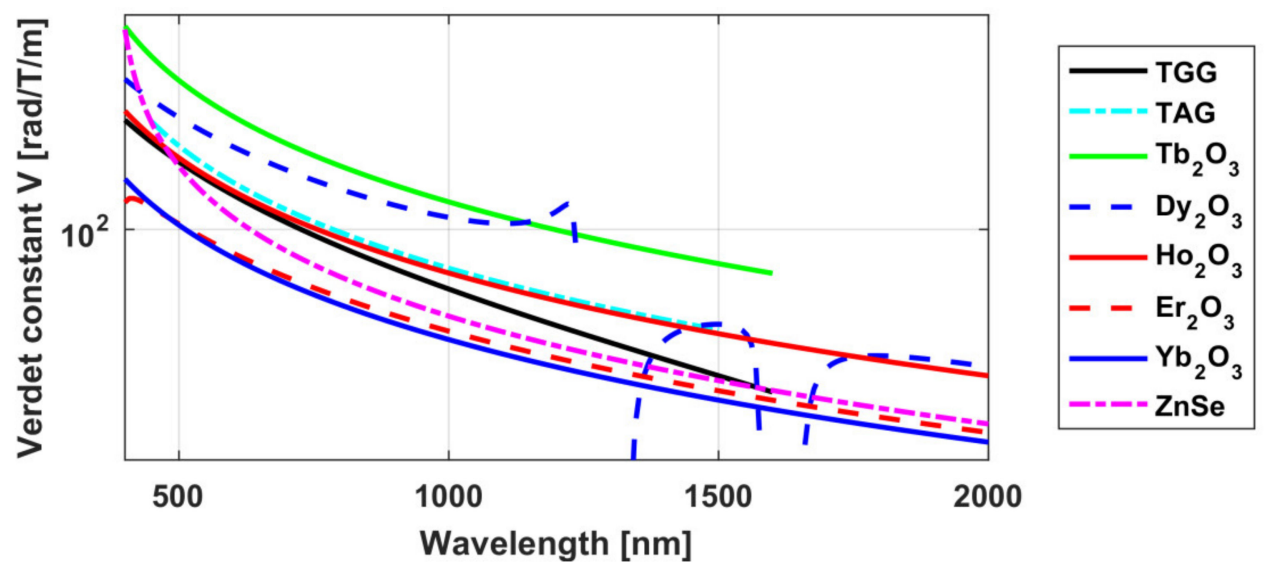

Figure 5. Dispersion trends of the Verdet constant of TGG [39], $\mathrm{Tb}_{2} \mathrm{O}_{3}[39,40]$, TAG [38,41], $\mathrm{Dy}_{2} \mathrm{O}_{3}$ [42], $\mathrm{Ho}_{2} \mathrm{O}_{3}$ [43,44], $\mathrm{Er}_{2} \mathrm{O}_{3}$ [45], $\mathrm{Yb}_{2} \mathrm{O}_{3}$ [46], ZnSe [47], and $\mathrm{Y}_{2} \mathrm{O}_{3}$ [42]).

The addition of sintering additives with absorption bands in the visible and near-IR regions of the spectrum ensures a significant increase of absorption in TAG ceramics. Measurements of thermally induced effects $[25,41,48,49]$ show that the smallest depolarization degree is introduced by pure TAG and TAG doped with silicon and cerium ions that have no absorption bands in this region, despite the fact that the highest transparency is shown by the samples doped with zirconium oxide [50]. 
The thermally induced depolarization as a function of the radiation power for best samples of these ceramics of $L_{45}$ in a magnetic system with a field strength of $2.5 \mathrm{~T}$ is plotted in Figure 6. It is clear from the graph that $P_{\max }$ of the TAG ceramics is $\sim 1.5$ times higher than of the TGG ceramics.

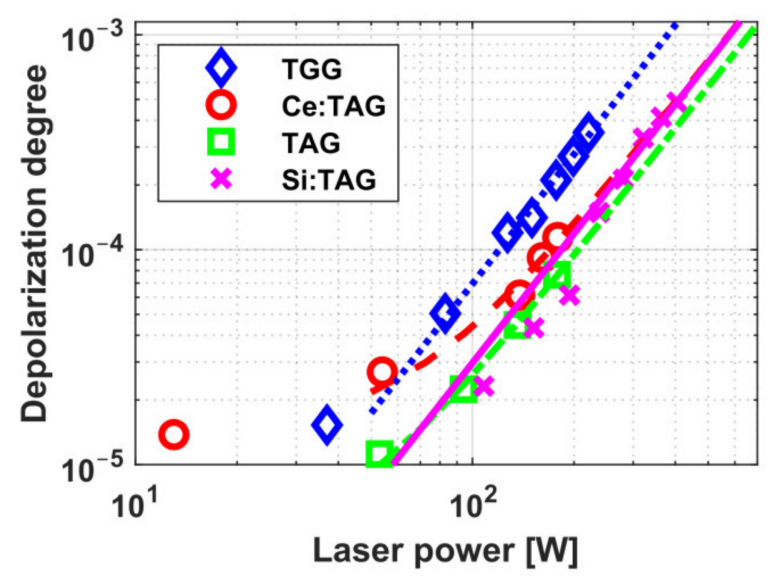

Figure 6. Depolarization degree versus laser radiation power in FI based on TGG, TAG, Si:TAG, and Ce:TAG ceramics.

The dependences of the optical power of thermal lens $D$ on the radiation power reported in $[25,48,49]$ for TAG and Ce $(0.1 \%)$ :TAG re-calculated for the length $L=L_{45}$ and beam diameter of $2.4 \mathrm{~mm}$ are shown in Figure 2. The value of $D$ is approximately equal for TAG and TGG, but is about 2 times higher for Ce:TAG, as the absorption of the sample with the content of cerium of $0.1 \%$ is higher than of the sample with $0.05 \%$.

With the use of magneto-optical ceramics we have developed a FI with an isolation ratio of $38 \mathrm{~dB}$ for a radiation power of $300 \mathrm{~W}$ and a focal length of the thermal lens of about $8 \mathrm{~m}$ [48]. The maximum power $P_{\max }$ was estimated to be $>700 \mathrm{~W}$. For an FI based on Ce:TAG we took a sample with a cerium content of $0.1 \%$, i.e., with the absorption being higher than in the case of an optimal cerium content of $0.05 \%$, and obtained only $31 \mathrm{~dB}$ at a radiation power of $300 \mathrm{~W}$. The use of the internal scheme of compensation of thermally induced depolarization allowed us to increase the isolation ratio up to $39 \mathrm{~dB}$ for the same power [49] and to obtain $P_{\max }>2 \mathrm{~kW}$.

Cryogenic cooling enables an additional reduction of thermally induced distortions and shortening of MAE length due to an increased Verdet constant. On cooling, the Verdet constant $V$ in TAG ceramics increases faster than $1 / T$, and at $89 \mathrm{~K}$ it amounts to $\mathrm{V}(1064 \mathrm{~nm})=221 \mathrm{rad} / \mathrm{T} / \mathrm{m}$ [51]. The temperature dependence of $V$ is plotted in Figure 7 . The thermally induced depolarization in a TAG ceramics sample reduces by more than 5 times, and the optical power of the thermal lens by more than 2 times as a result of cooling from 295 to $79 \mathrm{~K}$. In view of the increase of the Verdet constant at cooling to $79 \mathrm{~K}$, in a magnetic cell with a field strength of $1.9 \mathrm{~T}$ it is sufficient to use an element with a length of $2.3 \mathrm{~mm}$ to obtain, according to the estimates, $P_{\max }>3 \mathrm{~kW}$. However, due to the high scattering in the sample at cooling, the depolarization increases and at cooling to $150 \mathrm{~K}$, $P_{\text {max }}=0.5 \mathrm{~kW}[51,52]$.

Note that in the majority of our TAG ceramics samples we observed strong additional heating caused by scattering in the samples, which demanded active cooling of the MAE. Thus, being superior to TGG in the Verdet constant, TAG ceramics, after further improvement of its optical properties, can be successfully used in isolators for lasers with high average power. 


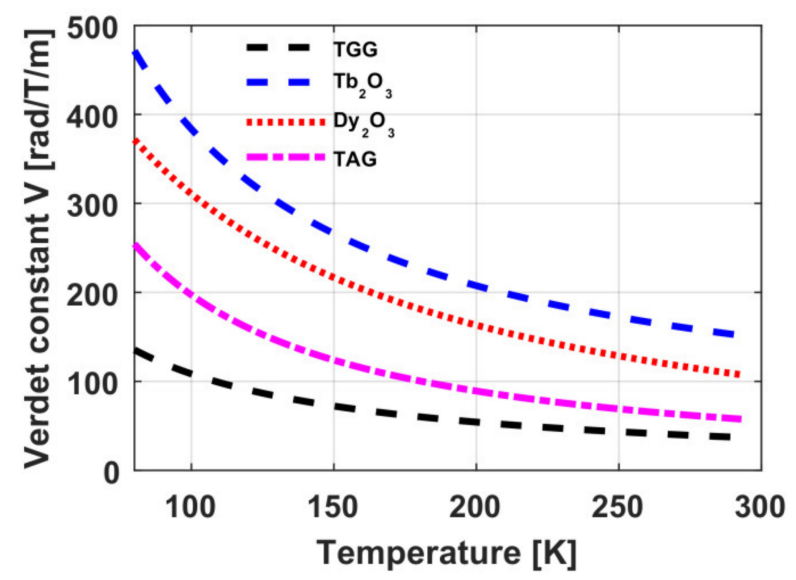

Figure 7. Verdet constant as a function of temperature at wavelength of $1064 \mathrm{~nm}$ : TGG $[18,23]$, TAG [51], $\mathrm{Tb}_{2} \mathrm{O}_{3}$ [53], $\mathrm{Dy}_{2} \mathrm{O}_{3}$ [54].

\subsection{Sesquioxide Ceramics}

The prospects of using sesquioxide ceramics thanks to their thermo-optical properties arose the interest of researcher more than 50 years ago [55]. We pay great attention to manufacturing [46,56-60], as well as to studying $[39,40,42,43,54,57]$ magneto-optical and thermo-optical properties of the sesquioxide ceramics $\mathrm{Y}_{2} \mathrm{O}_{3}, \mathrm{~Tb}_{2} \mathrm{O}_{3}, \mathrm{Dy}_{2} \mathrm{O}_{3}, \mathrm{Ho}_{2} \mathrm{O}_{3}, \mathrm{Er}_{2} \mathrm{O}_{3}$, and $\mathrm{Yb}_{2} \mathrm{O}_{3}$, that will be considered in this section.

\subsubsection{Production Technique}

To date, many methods have been developed for both the synthesis of initial rare-earth oxide nanopowders and methods for their subsequent consolidation into optical-quality ceramics. In our works, we use the method of self-propagating high-temperature synthesis (SHS) to obtain nanopowders containing matrix elements uniformly distributed over the volume, sintering and auxiliary additives $\left(\mathrm{LiF}, \mathrm{ZrO}_{2}\right.$, and rare earth oxides other than the main matrix oxide) $[58,60]$. SHS consists in the synthesis of a precursor containing nitrates of the corresponding metals and organic fuel-acetic or aminoacetic acids, followed by thermal initiation of its combustion. In the course of the reaction, the precursor melts and it is foamed by the evolved gases. The walls of this foam are one or tens of nanometers thick and consist of metal oxides. Fast combustion (a few seconds) and a significant volume of evolved gases prevent the particles from sintering with each other. Therefore, the foam crumbles to submicron particles even on insignificant mechanical impact (pressing, grinding, ultrasonic dispersion, etc.). Depending on the initial components and the features of SHS, the specific surface area of oxide powders of rare-earth materials (REE) varies from 30 to $100 \mathrm{~m}^{2} / \mathrm{g}$. The advantages of the method are the simplicity and technological flexibility of the process, which allows synthesizing mixed compositions with a homogeneous distribution of components, a small number of stages limiting the contamination of materials, and a high sinterability of the resulting powders. The main drawbacks of the method are the presence of inhomogeneous combustion areas (for example, near the walls), where rigid agglomerates can form, as well as a predominantly lamellar shape of the particles and a low bulk density of the powders, which hinder the formation of compacts with a homogeneous pore structure. The latter, among other things, imposes restrictions on the methods of their subsequent consolidation. So, without additional processing of powders (annealing and grinding), the achievable maximal thickness of laser-quality ceramics obtained by free vacuum sintering does not exceed 1-2 $\mathrm{mm}$. Thicker specimens can be obtained by hot pressing, in which the applied uniaxial pressure provides additional driving force to the consolidation.

The sinterability of the individual oxides is usually insufficient for producing laserquality ceramics without hot isostatic pressing and is a nontrivial task, even with its application. Therefore, at free vacuum sintering, a sintering additive $\mathrm{La}_{2} \mathrm{O}_{3}[45,58,61]$ and 
in some cases $\mathrm{Y}_{2} \mathrm{O}_{3}$ is used, when the solubility of lanthanum oxide is insufficient for a single-phase ceramics to be formed (for example, in $\mathrm{Dy}_{2} \mathrm{O}_{3}[42,56]$ ). These additives are isovalent to REE oxides and, except for some decrease in thermal conductivity, have do not negatively affect the characteristics of ceramics. At hot pressing, lithium fluoride is the most effective sintering additive [40]. It significantly activates the diffusion processes and is almost completely removed from the ceramics at the stage of open porosity. Thus, it has no impact on the final characteristics of the ceramics, except for some increase in the average grain size.

After sintering, an oxygen deficiency is usually formed in the ceramics, which gives them a dark shade and markedly reduces the transmittance in the visible and near-IR wavelength ranges. Therefore, before optical processing, the ceramics are annealed in air at temperatures of about $900{ }^{\circ} \mathrm{C}$ for $5 \mathrm{~h}$. The only exception is the ceramics based on terbium oxide, which is oxidized to $\mathrm{Tb}_{4} \mathrm{O}_{7}$ in air [59] and becomes opaque in the entire optical range.

\subsubsection{Results of the Studies}

Samples of magneto-active ceramics were studied in detail in the following works: $\mathrm{Tb}_{2} \mathrm{O}_{3}[39,40,53], \mathrm{Dy}_{2} \mathrm{O}_{3}[42,54,62], \mathrm{Ho}_{2} \mathrm{O}_{3}$ [44,45], $\mathrm{Er}_{2} \mathrm{O}_{3}$ [57], $\mathrm{Yb}_{2} \mathrm{O}_{3}$ [46], and $\mathrm{Y}_{2} \mathrm{O}_{3}$ [42]. The research [44] was made in collaboration with the Chinese colleagues of the Shanghai Institute of Ceramics. The theoretical dependences of the Verdet constant on wavelength obtained by approximating the experimental data of the above-mentioned works using the expression (9) in comparison with the widely used TGG material are presented in Figure 5 [63]. The approximation of the Verdet constant of yttria ceramics was obtained based on the data from [42] with the approximation coefficients $C=2.07 \times 10^{6}$ ( $\mathrm{rad} \times$ $\left.\mathrm{nm}^{2}\right) /(\mathrm{T} \times \mathrm{m})$ and $\lambda_{0}=232 \mathrm{~nm}$ from the expression (9). It is worth noting that these approximations do not describe the behavior of the Verdet constant in the regions with absorption, but give its good description in the wavelength ranges in which experiments were conducted in the above works.

The Verdet constant of undoped ceramics $\mathrm{Y}_{2} \mathrm{O}_{3}$ has a $\sim 20$ times lower absolute value than TGG [42]. The values of the Verdet constant of $\mathrm{Yb}_{2} \mathrm{O}_{3}$ and $\mathrm{Er}_{2} \mathrm{O}_{3}$ are close and are $\sim 2$ times less than of TGG in the range of its operation (up to $\sim 1.4 \mu \mathrm{m}$ ). A distinguishing feature of $\mathrm{Yb}_{2} \mathrm{O}_{3}$ and $\mathrm{Er}_{2} \mathrm{O}_{3}$ ceramics is the absence of absorption lines in the 1.1-6 $\mu \mathrm{m}$ and 1.7-7 $\mu \mathrm{m}$ ranges, respectively, so their application as a magneto-active material is promising only in the small range of $1.7-1.9 \mu \mathrm{m}$.

As the other ceramics $\left(\mathrm{Tb}_{2} \mathrm{O}_{3}, \mathrm{Dy}_{2} \mathrm{O}_{3}, \mathrm{Ho}_{2} \mathrm{O}_{3}\right)$ have a much larger Verdet constant and surpass even TGG, they are interesting as potential candidates throughout their transparency ranges/windows. $\mathrm{Tb}_{2} \mathrm{O}_{3}$ ceramics, like all $\mathrm{Tb}$-containing materials have a multiple absorption bands, starting at about 1.3-1.4 $\mu \mathrm{m} ; \mathrm{Dy}_{2} \mathrm{O}_{3}$ has several absorption lines in the 1-1.9 $\mu \mathrm{m}$ and 2.3-3.5 $\mu \mathrm{m}$ ranges, and $\mathrm{Ho}_{2} \mathrm{O}_{3}$ in the $1.07-1.3 \mu \mathrm{m}$ and $1.65-2.2 \mu \mathrm{m}$ ranges. The most attractive recommended ranges of the considered ceramics are clearly seen in the transmission spectra in Figure 8. In the range up to $\sim 1.4 \mu \mathrm{m}$, the most preferable materials are $\mathrm{Tb}_{2} \mathrm{O}_{3}$ and TAG (partly $\mathrm{Dy}_{2} \mathrm{O}_{3}$ ); in the 1.5-2.1 $\mu \mathrm{m}-\mathrm{Dy}_{2} \mathrm{O}_{3}$ or $\mathrm{Ho}_{2} \mathrm{O}_{3}$ (at shorter or longer wavelengths $\lambda$, respectively); in the 2.1-3.2 $\mu \mathrm{m}$ range $\mathrm{Ho}_{2} \mathrm{O}_{3}$ is the leader (due to transmittance); in the $\sim 3.2-6 \mu \mathrm{m}$ range $\mathrm{Dy}_{2} \mathrm{O}_{3}$ is preferable; and in the $6-8 \mu \mathrm{m}$ interval $\mathrm{Tb}_{2} \mathrm{O}_{3}$ takes the leadership again (thanks to the large Verdet constant).

Cryogenic cooling is a possible way to increase the Verdet constant of MAE made of a paramagnetic material [64], which can decrease the element length and, hence, increase the magnitude of thermally induced distortions. The theoretical dependences of the Verdet constant on temperature for $\mathrm{Tb}_{2} \mathrm{O}_{3}$ and $\mathrm{Dy}_{2} \mathrm{O}_{3}$ ceramics obtained in our studies $[53,54]$ are given in Figure 7. According to our results, on cooling, the Verdet constant increases in proportion to the inverse temperature. 


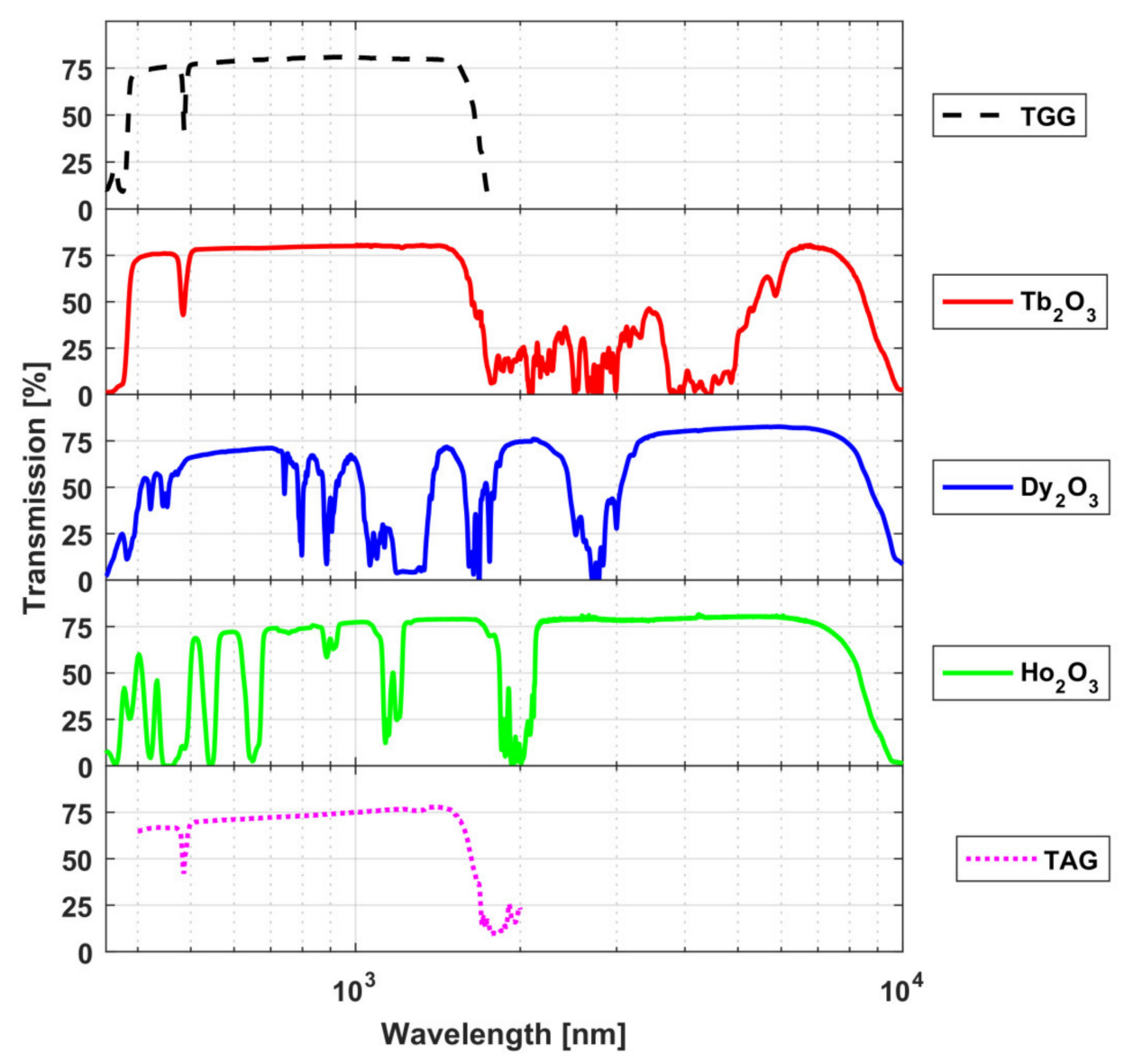

Figure 8. Recommended ranges of transmission spectra: TGG [39], $\mathrm{Tb}_{2} \mathrm{O}_{3}[39,40], \mathrm{Dy}_{2} \mathrm{O}_{3}[42]$, $\mathrm{Ho}_{2} \mathrm{O}_{3}[43,44]$, TAG.

\subsection{Zinc Selenide}

Zinc selenide (ZnSe) obtained by chemical vapor deposition (CVD) has found the widest application in power optics [65]. Its distinguishing feature is the optical transmittance close to the theoretical limit, high chemical and phase purity, weak mechanical stresses and small deviations from the stoichiometry of the components, as well as the possibility of manufacturing large-size elements. The CVD process runs in a graphite flow reactor with a square or rectangular cross section. Zinc and hydrogen selenide vapors with argon carrier gas are fed into the reactor, where their chemical interaction takes place. ZnSe is deposited on graphite substrates, and the resulting hydrogen is continuously removed with argon by a vacuum pump. Typical temperatures in the reactor are $700-750{ }^{\circ} \mathrm{C}$, the absolute pressure is about $0.5-5 \mathrm{kPa}$, the deposition rate is $2-2.5 \mathrm{~mm} /$ day, the area of one graphite substrate can reach $2 \mathrm{~m}^{2}$ with a layer thickness of tens of $\mathrm{mm}$. The resulting $\mathrm{ZnSe}$ sheets are removed from the substrate, cut into elements, and optically processed.

During the process, the initial zinc and selenium undergo a number of physical and chemical transformations that ensure effective purification to give the purity of CVD$\mathrm{ZnSe}$ of $4 \mathrm{~N}$ or more. The synthesis temperature is less than half the melting point of the material. This makes it possible to reduce the intake of impurities from the materials of the equipment, maintain a relatively low average grain size of $20-80 \mu \mathrm{m}$, significantly reduce the solubility of superstoichiometric zinc or selenium in the matrix, and, hence, reduce the probability of secondary phase formation when $\mathrm{ZnSe}$ is cooled to room temperature, as well as reduce mechanical stresses due to the absence of phase transitions during cooling. The disadvantages of the CVD method are its technological complexity, low material growth rate, high toxicity and explosion-fire hazard of intermediate compounds, and 
the yield of the laser-quality target material rarely exceeding $25 \%$ in terms of the initial components.

We measured the dispersion dependence of the Verdet constant $V(\lambda)$ of polycrystalline CVD-ZnSe at room temperature in the range of 532-1940 nm in the work [47]. The diamagnetic rotation of the polarization plane was observed, which was opposite to that of paramagnetic crystals (i.e., the sign of the Verdet constant of ZnSe was positive). The experimental dependence is well approximated by the expression (9). For $C=2.55 \cdot 10^{7} \mathrm{rad} \cdot \mathrm{nm}^{2} /(\mathrm{T} \cdot \mathrm{m})$ and $\lambda_{0}=380 \mathrm{~nm}$, the approximation accuracy was $9 \%$. The graph of the approximated dependence is shown in Figure 5. At a wavelength of $1064 \mathrm{~nm}$, the Verdet constant of $\mathrm{ZnSe}$ was $28.3 \mathrm{rad} / \mathrm{T} / \mathrm{m}$, which is a little lower than that of TGG $(\sim 22 \%)$. Therefore, it might seem that this material is of the greatest interest for laser radiation at wavelengths over $\sim 1.5 \mu \mathrm{m}$, where TGG has high absorption and stops working. The Verdet constant at a wavelength of $1940 \mathrm{~nm}$ was $7.7 \mathrm{rad} / \mathrm{T} / \mathrm{m}$, which is a fairly high value. The combination with the optical transparency of ZnSe in this range made it possible to implement the corresponding FI. Thermally induced depolarization was not observed experimentally up to $20 \mathrm{~W}$ and according to the estimates, $P_{\max }$ was $>150 \mathrm{~W}$ [47]. The dependence of the polarization of the transmitted radiation on its power is shown in the graph in Figure 9 (circles and dash-dot line). The FI for $\lambda=1075 \mathrm{~nm}$ was also developed and investigated, with $P_{\max } \sim 350 \mathrm{~W}$ (Figure 9, diamonds and dashed curve).

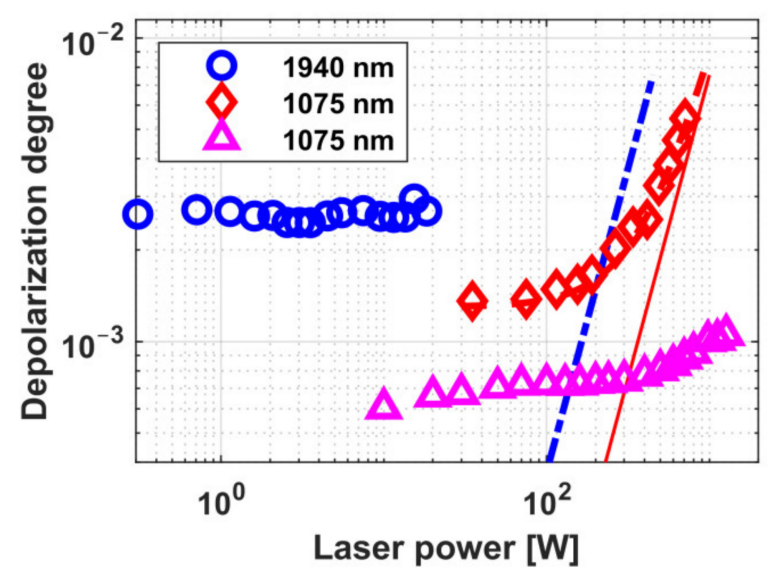

Figure 9. Power dependence of radiation depolarization in FIs based on CVD-ZnSe elements for a wavelength of $1940 \mathrm{~nm}$ (circles-experiment, dash-dot line-calculation [47]) and for a wavelength of $1075 \mathrm{~nm}$ (diamonds—experiment, dashed curve—calculation [47], triangles—experiment [47,66].

Note that we are just starting to work with chalcogenides and, believe that they are very interesting and promising materials for high-power FIs. The CVD-ZnSe samples under study were obtained in processes with different accuracy of controlling the reaction parameters. An isolator for the radiation wavelength of $1075 \mathrm{~nm}$ was also explored on the basis of a sample synthesized in the process with a more accurate maintenance of the stoichiometric ratio of the reaction components [66]. No thermally induced depolarization was observed in this FI at a radiation power up to $1270 \mathrm{~W}$ (Figure 9, triangles). It was proved that these polarization distortions would not be observed even at powers exceeding $2.5 \mathrm{~kW}$. The obtained result makes high-purity CVD-ZnSe one of the most promising materials for creating Faraday isolators with a multikilowatt power operating at room temperature.

\subsection{Spinel}

\subsubsection{Production Technique}

The ceramics of magnesium aluminate $\left(\mathrm{MgAl}_{2} \mathrm{O}_{4}\right)$ and zinc aluminate $\left(\mathrm{ZnAl}_{2} \mathrm{O}_{4}\right)$ spinels are of the greatest interest for laser applications. They have high thermal conductivity (about $18 \mathrm{~W} / \mathrm{m} / \mathrm{K}$ at $300 \mathrm{~K}$ [67]), microhardness ( $H_{\mathrm{V}}$ about 16 [68], and $11 \mathrm{GPa}$ [69], 
respectively), a wide range of transparency (0.2-6 and 0.3-7 $\mu \mathrm{m}$, respectively), radiation resistance, and an isotropic structure.

An approach to the synthesis of stoichiometric $\mathrm{MgAl}_{2} \mathrm{O}_{4}$ nanopowders by means of hydrolysis of double magnesium-alumininate isopropoxide $\mathrm{MgAl}_{2}\left(\mathrm{OPr}^{i}\right)_{8}$ with subsequent annealing of the formed hydroxides in air at a temperature of $700-900{ }^{\circ} \mathrm{C}$ was proposed in $[68,70]$. Double magnesium-aluminate isopropoxide has a relatively high volatility, and a particularly pure precursor of spinel nanopowders can be obtained from raw materials of technical qualification by the method of single vacuum distillation [71]. A sintering LiF $3 \mathrm{wt} \%$. additive was introduced at the hydrolysis stage to ensure its uniform distribution in the powder. This evidently explains the relatively small grain growth after consolidation (the main mode was from 2.5 to $20 \mu \mathrm{m}$ ) compared to the results of the studies where lithium fluoride was introduced into spinel powder by ball milling [72]. Hot pressing of the powders was done in a graphite mold at a temperature of $1600{ }^{\circ} \mathrm{C}$ with an soaking time of $1 \mathrm{~h}$. $\mathrm{MgAl}_{2} \mathrm{O}_{4}$ ceramics with a thickness of $1 \mathrm{~mm}$ had an optical transmittance of $83.7 \%$ at a wavelength of $1100 \mathrm{~nm}$.

Zinc and aluminum isopropylates do not form volatile double alkoxides; therefore, we have developed a somewhat different method for the synthesis of $\mathrm{ZnAl}_{2} \mathrm{O}_{4}$ powder [73]. It consisted in the hydrolysis of aluminum isopropoxide with an aqueous solution of zinc formate, followed by drying and calcination of the resulting sol at a temperature of $700{ }^{\circ} \mathrm{C}$ for $20 \mathrm{~min}$. Up to $6 \%$ mass of zinc fluoride was added to the initial solution as a sintering additive. The powder was consolidated into transparent ceramics by hot pressing at $1520{ }^{\circ} \mathrm{C}$ for $4 \mathrm{~h}$. $\mathrm{ZnAl}_{2} \mathrm{O}_{4}$ ceramics with a thickness of $4 \mathrm{~mm}$ had an optical transmittance of about $83 \%$ at a wavelength of $1100 \mathrm{~nm}$.

For doping spinels at the stage of hydrolysis, nitrates of transition metals, including rare-earth metals, were introduced. Ions of "light" elements, such as $\mathrm{Ti}, \mathrm{Cr}, \mathrm{Fe}, \mathrm{Co}$, and $\mathrm{Ni}$ have a high solubility in spinels and practically did not reduce the optical transmittance of ceramics outside their absorption lines, see, for example [73,74]. Whereas the introduction of up to $0.5 \%$ mol. rare earth oxides, including Sc and Y, led to high scattering in the spinels due to the formation of secondary phases of the corresponding aluminates [75].

\subsubsection{Results of the Studies}

In this section we consider the results that have not been published before. We investigated the spectral dependences of the Verdet constant of magnesium-aluminate (including with various dopants such as $\mathrm{Sc}, \mathrm{Ti}, \mathrm{Tb}, \mathrm{Ce}$, and $\mathrm{Yb}$ ) and zinc-aluminate spinels in the VIS-IR wavelength range (405-1064 nm), that are presented in Figure 10.

The undoped spinel demonstrates diamagnetic rotation of the plane of polarization. Its spectral dependence can be approximated by the expression (9) to an accuracy better than $3.2 \%$ with the coefficients $C=2.47 \cdot 10^{6} \mathrm{rad} \cdot \mathrm{nm}^{2} /(\mathrm{T} \cdot \mathrm{m})$ and $\lambda_{0}=208 \mathrm{~nm}$. At the wavelength of $1064 \mathrm{~nm}$, spinel is $\sim 15$ times inferior to TGG in terms of the Verdet constant.

As can be seen from Figure 10, doping of the spinel (with all the listed above ions except $\mathrm{Yb}$ ) in the considered concentrations does not lead to a significant change in the Verdet constant. The deviations from the dispersion dependence, corresponding to the undoped spinel, can be explained by the measurement error same as in the case of doped TAG ceramics. More significant deviations observed for Yb:spinel can be attributed to the presence of absorption lines of the $\mathrm{Yb}^{3+}$ ion in the considered wavelength range, the proximity to which can significantly change the form of the dispersion dependence, as in the case of $\mathrm{Dy}_{2} \mathrm{O}_{3}$ ceramics [62], when doping significantly degrades the optical quality of the medium, reduces transmittance, and increases the scattering of transmitted radiation.

Thermally induced depolarization was measured in a sample of undoped magnesiumaluminate spinel. The inset in Figure 10 shows the measurement results for a 3-mm thick sample re-calculated for the length $L_{45}$ in the case of a magnetic field with a strength of $2.5 \mathrm{~T}$. Thermally induced depolarization is expected to provide an isolation ratio of $>30 \mathrm{~dB}$ in such an FI with a maximal radiation power $P_{\max }$ of $\sim 15 \mathrm{~W}$ only. 


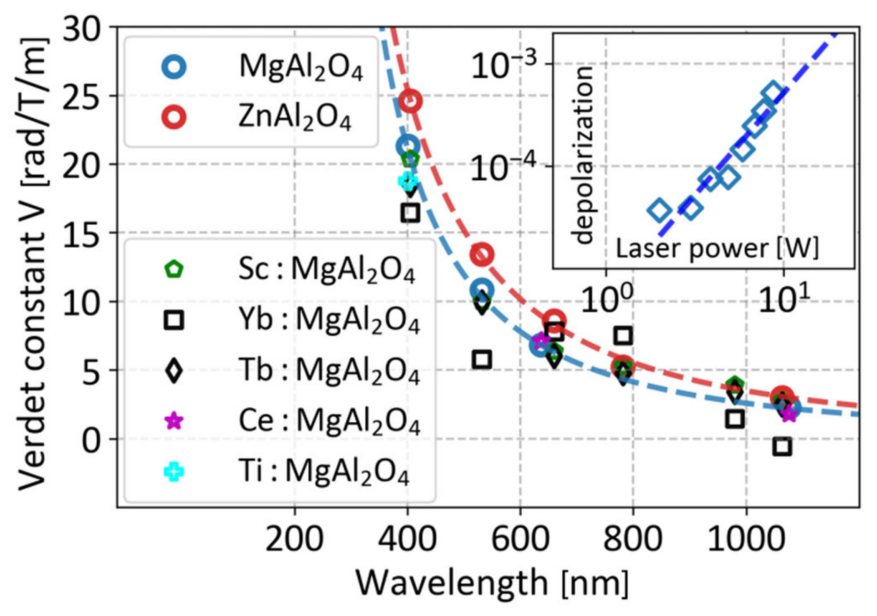

Figure 10. Spectral dependence of the Verdet constant in spinel samples: $\mathrm{MgAl}_{2} \mathrm{O}_{4}$ (red circles), $\mathrm{ZnAl}_{2} \mathrm{O}_{4}$ (blue circles), $\mathrm{Sc}(0.2 \%): \mathrm{MgAl}_{2} \mathrm{O}_{4}$ (pentagons), $\mathrm{Yb}(0.5 \%): \mathrm{MgAl}_{2} \mathrm{O}_{4}$ (squares), $\mathrm{Tb}(0.1 \%): \mathrm{MgAl}_{2} \mathrm{O}_{4}$ (diamonds), $\mathrm{Ce}(0.5 \%): \mathrm{MgAl}_{2} \mathrm{O}_{4}$ (asterisks), $\mathrm{Ti}(1.8 \%): \mathrm{MgAl}_{2} \mathrm{O}_{4}$ (plus signs). Dashed curves correspond to the approximation of dispersion dependences for samples of undoped spinels. Inset: thermally induced depolarization as a function of laser radiation power in a sample of magnesium-aluminate spinel re-calculated for the length $L_{45}$.

Zinc-aluminate spinel also exhibits diamagnetic rotation of the plane of polarization. Its spectral dependence can be approximated by the expression (9) to an accuracy of $\sim 10 \%$ with the values of the coefficients $C=3.39 \cdot 10^{6} \mathrm{rad} \cdot \mathrm{nm}^{2} /(\mathrm{T} \cdot \mathrm{m})$ and $\lambda_{0}=163 \mathrm{~nm}$, i.e., its Verdet constant is $15-30 \%$ higher than the Verdet constant of $\mathrm{MgAl}_{2} \mathrm{O}_{4}$ in the investigated wavelength range.

Thus, the magneto-optical characteristics of both types of spinel are significantly inferior to those of the popular magneto-optical materials. This is the main limitation of its use in magneto-optical devices at the moment.

\section{Laser/Active Ceramics}

$\mathrm{Yb}: Y A G$ is perhaps most often used for creating lasers with high average and peak power because of the optimal combination of laser (long lifetime and gain cross section) and material (high thermal conductivity) characteristics, as well as its low cost [76-80]. Works aimed at creating pulse-periodic Yb:YAG lasers with high average and peak power for scientific and industrial applications are underway is different countries: HiLASE (Dolni Brezany, the Czech Republic), Genbu (Osaka, Japan), DiPOLE (Chilton, UK) [81-83], and others. However, the aperture of the available single-crystal elements is insufficient to obtain the output energy of a few to tens of Joules.

Quite a number of other factors should be taken into consideration concerning the use of Yb:YAG. A serious problem arises in disk laser amplifiers: due to the high gain in the medium, the energy stored in the AE is strongly limited by the effects of amplified spontaneous emission (ASE) and parasitic generation. During cryogenic cooling, there occur both a significant undesirable decrease in the width of the gain spectrum and a shift of its center wavelength $\lambda_{\max }$. At a temperature of $80 \mathrm{~K}$, the spectrum width is comparable to the shift of $\lambda_{\max }$. Therefore, for a cryogenic Yb:YAG amplifier, it is necessary either to artificially shift the lasing wavelength of the Yb:YAG generator operating at room temperature, or to cool the crystal of the Yb:YAG generator. In this case, the duration of the output pulses is limited to $\sim 2 \mathrm{ps,} \mathrm{which} \mathrm{is} \mathrm{too} \mathrm{long} \mathrm{for} \mathrm{a} \mathrm{number} \mathrm{of} \mathrm{applications.}$ One of the options for solving these problems is to use a medium with a lower emission cross section than that of Yb:YAG to suppress the ASE effect and with a spectrum that enables operation at cryogenic temperatures. To date, the commonly used broadband media $\mathrm{Yb}: \mathrm{KYW}, \mathrm{Yb}: \mathrm{KGW}$, and $\mathrm{Yb}: \mathrm{CaF}_{2}$ working with ultrashort pulses [84-86] fit this description. However, in $\mathrm{Yb}: \mathrm{CaF}_{2}$, the emission cross section is too small to efficiently 
extract the energy stored in the active element, and the thermal conductivity of $\mathrm{Yb}: \mathrm{KYW}$ and $\mathrm{Yb}: \mathrm{KGW}$, that is lower than that of $\mathrm{Yb}: \mathrm{YAG}$, limits the average output power.

Thus, to increase the output energy and efficiency of modern disk lasers it is necessary to develop and implement large-aperture AE technology, as well as to identify new active laser media with a more suitable gain spectrum and high thermal conductivity. A promising approach to solving these problems is to use optical laser ceramics. The currently available technologies make it possible to manufacture large-aperture elements tens of centimeters in diameter [87] and to create active elements from media that are hard or impossible to grow in the form of large single crystals. As was mentioned above, examples of such media are the ceramics of rare-earth-metal sesquioxides $\mathrm{Yb}: \mathrm{Y}_{2} \mathrm{O}_{3}, \mathrm{Yb}: \mathrm{Lu}_{2} \mathrm{O}_{3}$ and $\mathrm{Yb}: \mathrm{Sc}_{2} \mathrm{O}_{3}$ whose thermal conductivity is higher and gain spectrum is wider than in Yb:YAG [88-90]. As the gain is lower than in $\mathrm{Yb}: \mathrm{YAG}$, the energy is more effectively stored in the AE. As the gain is higher than in $\mathrm{Yb}: \mathrm{CaF}_{2}$, the stored energy can be extracted more effectively. The thermal conductivity that is higher than in $\mathrm{Yb}: \mathrm{KYW}$ and $\mathrm{Yb}: \mathrm{KGW}$ enables broadband radiation gain at a higher average power. In addition, as will be shown below, the central wavelength and gain bandwidth of $\mathrm{Yb}: \mathrm{Y}_{2} \mathrm{O}_{3}$ and $\mathrm{Yb}: \mathrm{Lu}_{2} \mathrm{O}_{3}$ allow using such a cryogenic amplifier together with the available water-cooled master oscillator based on Yb:YAG crystals.

\subsection{Yb:YAG Ceramics}

In contrast to rare-earth oxides, self-propagating high-temperature synthesis (SHS) of YAG powders does not ensure production of ceramics with high optical transparency [91]. A possible reason for this is the contamination of powders with carbon [92], the removal of which requires calcination at high temperatures, leading to agglomeration of powders and a decrease in their sinterability. Consequently, we used an alternative method for their preparation-the sol-gel synthesis [93]. A method for preparing powders from a mixture of yttrium hydroxoacetate sols [94] and boehmite by spray drying [95], as well as direct sintering of compacted amorphous xerogel from a mixture of aluminum hydroxonitrate sols [96] and yttrium hydroxoacetate [97] was developed. Ceramics with high optical transparency were produced by free vacuum sintering at a temperature of $1760{ }^{\circ} \mathrm{C}$ for $2 \mathrm{~h}$, followed by annealing in air at $1300^{\circ} \mathrm{C}$ for $1 \mathrm{~h}$. These methods can be effectively used for manufacturing active ceramic elements of disk configuration. In Russia, there are several scientific groups involved in the development and production of laser ceramics, including Yb:YAG ceramics. We also tested the ceramics manufactured at the Institute of Electrophysics of the Ural Branch of the Russian Academy of Sciences and the Ural Federal University (Yekaterinburg, Russia), according to the original method, with the nanopowders obtained by laser ablation [98].

Since Yb:YAG ceramics, as well as Yb:YAG crystals are popular and well-studied materials, we have not investigated them at room temperatures in ample detail and have not obtained significant results. Therefore, we will only give references to our works in which the laser properties of Yb:YAG ceramics were studied [99,100]. However, cryogenic cooling of active elements is known to be a significant advantage in the development of lasers with high average and high peak power, which improves both thermo-optical and laser characteristics.

Within the framework of collaborative research, we have obtained a number of samples of Yb:YAG ceramics with a diameter of $20 \mathrm{~mm}$, a thickness of $1.4 \mathrm{~mm}$ and 5 at.\% doping, manufactured at the AMRC LAB, Nanyang Technological University, Singapore. The ceramic samples were produced by the method of reaction sintering in vacuum at a temperature of $1770{ }^{\circ} \mathrm{C}$. Details of this technology are described in [101]. Depending on the duration of sintering at a temperature of $1770{ }^{\circ} \mathrm{C}$ varying from 8 to $40 \mathrm{~h}$, it is possible to obtain ceramics with an average grain size from 6 to $25 \mu \mathrm{m}$. Studies of three samples of different grain size showed that the behavior of their spectral characteristics on cooling is close to that observed in single crystals. Due to the large thickness of the samples, the lifetime was measured only at the liquid nitrogen temperature (when there was no re-absorption from the lower working laser level) and amounted to $~ 900 \mu \mathrm{s}$. 
Using the above samples, we developed a cryogenic Yb:YAG disk laser, in which the generated pulse with a duration of $70 \mathrm{~ns}$ and an energy of 3-5 mJ was amplified in a preamplifier to $54 \mathrm{~mJ}$ at a repetition rate of $200 \mathrm{~Hz}$. With the use of two disk AEs made of Yb:YAG ceramics in a multipass cryogenic amplifier (Figure 11) with a filling cooling system, we obtained $233 \mathrm{~mJ}$ in $70 \mathrm{~ns}$ pulses at a repetition rate of $143 \mathrm{~Hz}$ with a high $(20 \%)$ pump efficiency. These output parameters are the best among the currently developed cryogenic pulse-periodic laser systems based on thin disks made of Yb:YAG ceramics [102].
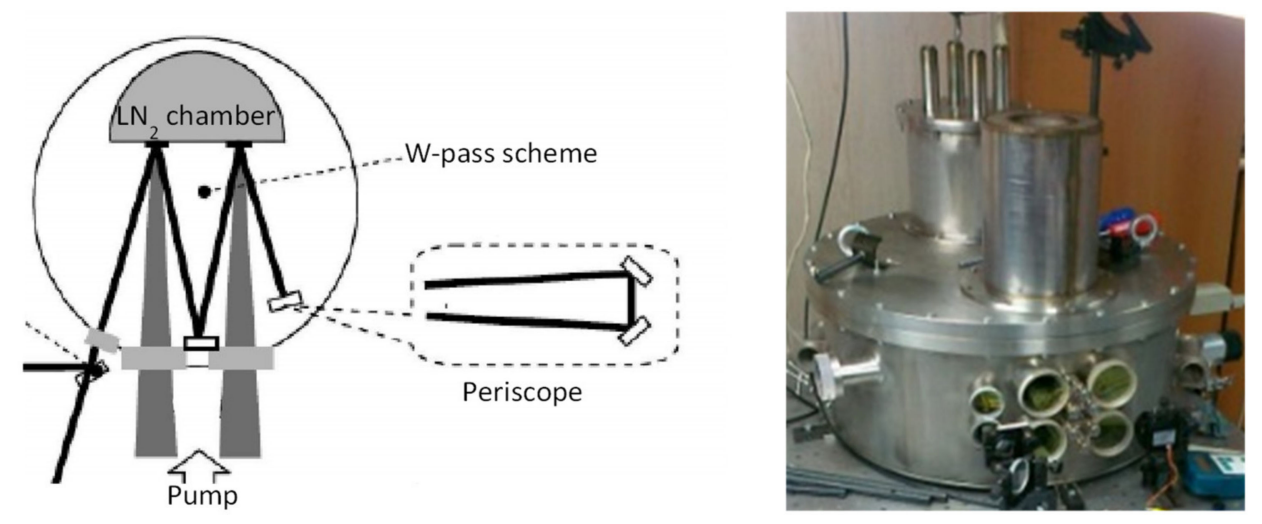

Figure 11. Optical scheme and a photograph of vacuum cryostat of a multipass cryogenic amplifier based on Yb:YAG ceramics.

\subsection{Sesquioxide Ceramics}

\subsubsection{Manufacturing and Spectral Properties}

The initial nanopowders of REE oxides were obtained by the SHS technique described in Section 2.3.1. Oxide powders were successfully synthesized on the basis of the $\mathrm{Sc}_{2} \mathrm{O}_{3}[103,104], \mathrm{Y}_{2} \mathrm{O}_{3}[105]$ and $\mathrm{Lu}_{2} \mathrm{O}_{3}[106,107]$ matrixes doped with active REE ions, the media doped with $\mathrm{Yb}^{3+}$ ions being of major interest. The powders were consolidated into laser ceramics by means of free vacuum sintering and hot pressing, as well as by a specially developed microwave sintering technique. The microwave sintering was performed in the working chamber of a gyrotron complex operating in a CW mode at a frequency of $24 \mathrm{GHz}$ with an automatically controlled microwave power of up to $6 \mathrm{~kW}$. The compacted powders were placed in the center of a quartz crucible, covered with granular yttrium oxide for thermal isolation, and heated in a residual air atmosphere at a pressure of about $10 \mathrm{~Pa}$ in a microwave field to a temperature of about $1720^{\circ} \mathrm{C}$. This method was successfully used to produce $\left(\mathrm{Yb}_{0.05} \mathrm{Y}_{0.85} \mathrm{La}_{0.1}\right)_{2} \mathrm{O}_{3}$ [108]. The laser quality was confirmed by the generation in a quasicontinuous mode. The differential efficiency with a maximum at a wavelength of $1033 \mathrm{~nm}$ was $25 \%[109,110]$.

We also tested the $\mathrm{Yb}: \mathrm{Y}_{2} \mathrm{O}_{3}$ ceramics manufactured at the Institute of Electrophysics of the of the Ural Branch of the Russian Academy of Sciences and the Ural Federal University (Yekaterinburg, Russia) [111]. Samples of the ceramics produced by these two methods were compared in the paper [112]. We attributed the difference in the generation efficiency to chemical purity of the ceramic elements that manifested itself by luminescence. Four groups of spectral lines with maxima at 409.6, 490, 550, and $662 \mathrm{~nm}$ were observed in the visible spectra of this material (Figure 12). The radiation in these wavelength ranges is usually associated with cooperative processes of the interaction of a $\mathrm{Yb}^{3+}$ ion with other REM ions (e.g., the radiation at $\sim 409, \sim 550,650-680 \mathrm{~nm}$ with $\mathrm{Yb}^{3+}-\mathrm{Er}^{3+}$, and at $490 \mathrm{~nm}$ with cooperative luminescence of $\mathrm{Yb}^{3+}-\mathrm{Yb}^{3+}$ clusters, and so on). Thus, prior to fabricating the active element, one can compare the intensity of luminescence in the visible range with the reference sample and, thus, obtain information on the spectral properties of the ceramic elements, in addition to their optical quality. 


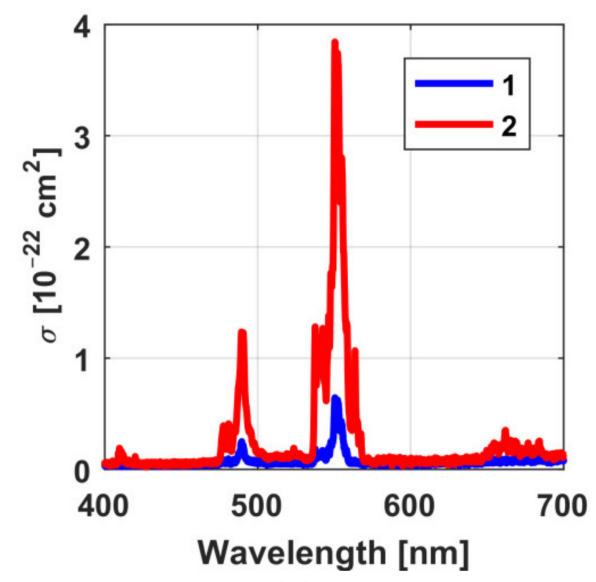

(a)

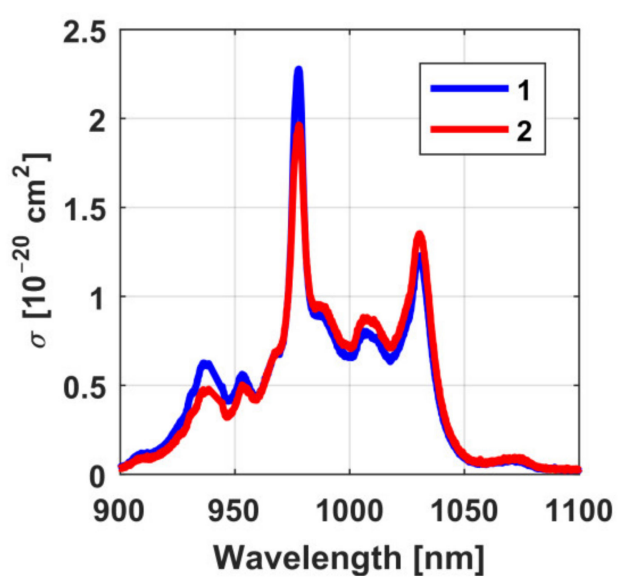

(b)

Figure 12. Luminescence spectra in (a) visible and (b) near-IR range of $\mathrm{Yb}: \mathrm{Y}_{2} \mathrm{O}_{3}$ ceramics samples.

The $\mathrm{Sc}_{2} \mathrm{O}_{3}$ material has high thermal conductivity $(18 \mathrm{~W} / \mathrm{m} / \mathrm{K}$ for an undoped material), low phonon energy, and large ground state splitting of doping ytterbium ions, which makes it a potential candidate to be used for $\mathrm{AE}$ lasers with high average power. $2 \%$ $\mathrm{Yb}: \mathrm{Sc}_{2} \mathrm{O}_{3}$ ceramics were produced from SHS-powders containing $1 \mathrm{wt} \%$ of $\mathrm{LiF}$ sintering additive by hot pressing at $1600{ }^{\circ} \mathrm{C}$ for $1 \mathrm{~h}$ [104]. Despite the relatively low temperature and sintering time, secondary grain re-crystallization was initiated having bimodal distribution with mode maxima at about 10 and $30 \mu \mathrm{m}$. The ceramics transmittance in the near-IR range was about $78 \%$, which is approximately $2 \%$ lower than the theoretical value. The maximum of the luminescence spectrum compared with the maximum of the $\mathrm{Yb}^{3+}$ ion luminescence in the YAG, $\mathrm{Y}_{2} \mathrm{O}_{3}$, and $\mathrm{Lu}_{2} \mathrm{O}_{3}$ matrices is shifted to the region of $\sim 1.04 \mu \mathrm{m}$ due to the larger splitting of the laser level. The width of the luminescence spectrum is $\sim 10 \mathrm{~nm}$, which will allow using this material to generate laser pulses with a duration of $\sim 100 \mathrm{fs}$. The laser quality was confirmed by lasing in a quasicontinuous mode. The differential efficiency with a maximum at a wavelength of $1040 \mathrm{~nm}$ was $24 \%$.

For the generation and amplification of ultrashort pulses, a laser material with a wide gain band is required. The gain band of pure sesquioxide materials is sufficient to generate and amplify pulses with a duration of $\sim 100 \mathrm{fs}$. To obtain a wider gain band using the same materials, several active elements from different materials or solid melts can be used. For example, the ceramics with this composition $\left(\mathrm{Lu}_{0.65} \mathrm{Y}_{0.25} \mathrm{La}_{0.05} \mathrm{Yb}_{0.05}\right)_{2} \mathrm{O}_{3}$ were manufactured by free vacuum sintering [113] at a temperature of $1750^{\circ} \mathrm{C}$. The spectral properties of solid solutions of sesquioxide ceramics were investigated in $[113,114]$. A number of the studied ceramics samples were also produced at the Institute of Electrophysics, Ural Branch of the Russian Academy of Sciences (Yekaterinburg, Russia) by means of solid-phase sintering of nanopowders of complex chemical composition. When some of the $\mathrm{Y}^{3+}$ cations were replaced by $\mathrm{Lu}^{3+}$ cations in the yttrium oxide matrix, the maxima of the luminescence bands of $\mathrm{Yb}^{3+}$ ions were shifted to the long-wavelength region of the spectrum from $1030 \mathrm{~nm}$ to $1032 \mathrm{~nm}$. Besides, the addition of $\mathrm{Lu}_{2} \mathrm{O}_{3}$ leads to broadening of the gain band profile from 15 to $18 \mathrm{~nm}$ at half maximum for the peak in the vicinity of $1032 \mathrm{~nm}$. The laser quality was confirmed by lasing on ceramic samples $\left(\mathrm{Lu}_{0.65} \mathrm{Y}_{0.25} \mathrm{La}_{0.05} \mathrm{Yb}_{0.05}\right)_{2} \mathrm{O}_{3}$ with a differential efficiency of $32.3 \%$ and a maximal average power of $10 \mathrm{~W}$.

\subsubsection{Studies of Thermo-Optical Characteristics}

We investigated the thermo-optical properties of $\mathrm{Y}_{2} \mathrm{O}_{3}, \mathrm{Lu}_{2} \mathrm{O}_{3}$, and $\mathrm{Sc}_{2} \mathrm{O}_{3}$ ceramics samples doped with $\mathrm{Yb}^{3+}$ ions with concentrations of $1.8 \%$, and $2.5 \%$, respectively [115], manufactured by Konoshima Chemical Co. Ltd. (Kagawa, Japan), a leader in the production of commercially available laser ceramics, and a sample of a Yb:YAG single crystal with an $\mathrm{Yb}$ concentration of $10 \%$. The analysis of the results showed that the $\mathrm{Lu}_{2} \mathrm{O}_{3}$ and $\mathrm{Sc}_{2} \mathrm{O}_{3}$ materials have close values of $Q_{e f f}$, while for $\mathrm{Y}_{2} \mathrm{O}_{3}$ this value is $30 \%$ lower. Since thermally 
induced depolarization is proportional to the square of $Q_{e f f} / \kappa$, this difference for the same heat release will lead to a more than twofold difference in integral depolarization degree. By comparing the values of $Q_{e f f}$ and taking into account the dependence of the thermal conductivity coefficient of these materials on the concentration of $\mathrm{Yb}^{3+}$ ions [116] we can say that for producing AEs with a high concentration of $\mathrm{Yb}^{3+}$ ions, $\mathrm{Lu}_{2} \mathrm{O}_{3}$ ceramics is more beneficial in terms of minimization of thermally induced polarization distortions, as for the concentrations $>4$ at. $\%$, thermally induced depolarization in this material will be less than in $\mathrm{Y}_{2} \mathrm{O}_{3}$ and $\mathrm{Sc}_{2} \mathrm{O}_{3}$, other conditions being equal. Contrariwise, for AEs with a low $\mathrm{Yb}^{3+}$ ion concentration, $\mathrm{Sc}_{2} \mathrm{O}_{3}$ ceramics is more beneficial, as its value of $Q_{e f f}$ only $15 \%$ less, whereas the thermal conductivity coefficient increases with decreasing $\mathrm{Yb}^{3+}$ concentration much faster than in $\mathrm{Y}_{2} \mathrm{O}_{3}$. For the $\mathrm{Yb}^{3+}$ concentrations lower than 1 at.\%, the thermally induced depolarization arising in $\mathrm{Sc}_{2} \mathrm{O}_{3}$ will be less than in $\mathrm{Y}_{2} \mathrm{O}_{3}$ or $\mathrm{Lu}_{2} \mathrm{O}_{3}$, other conditions being equal.

Analogously, in Ref. [115] we compared the optical powers of thermal lenses of the above ceramics. Comparison of the thermo-optical characteristic $P$ singles out the $\mathrm{Sc}_{2} \mathrm{O}_{3}$ material, in which this value is significantly lower than in $\mathrm{Y}_{2} \mathrm{O}_{3}$ and $\mathrm{Lu}_{2} \mathrm{O}_{3}$. Like for polarization distortions, according to Equation (1), $D$ is inversely proportional to the thermal conductivity coefficient, which itself is a function of the concentration of the $\mathrm{Yb}^{3+}$ ion. For $\mathrm{Sc}_{2} \mathrm{O}_{3}$, the $P / \kappa$ value is less than for $\mathrm{Y}_{2} \mathrm{O}_{3}$ and $\mathrm{Lu}_{2} \mathrm{O}_{3}$ in the entire range of $\mathrm{Yb}^{3+}$ concentrations presented in Ref. [116]. The lower the thermo-optical characteristic $P$, the lower the optical power of the thermal lens introduced by the volume of the optical element is.

The thermo-optical properties of $\mathrm{Ho}_{2} \mathrm{Y}_{2} \mathrm{O}_{3}(0.5,1$, and 2 at.\% Ho) ceramic samples manufactured at the Kotelnikov Institute of Radio Engineering and Electronics (Moscow, Russia) were investigated in the work [117]; the dependence of the thermal conductivity coefficient on the concentration of holmium ions was measured using phase-shifting interferometry [118]. The thermal conductivity coefficient of the samples under study correlated with the concentration of $\mathrm{Ho}^{3+}$ ions; the higher the concentration, the lower the thermal conductivity coefficient, which decreased by $\sim 11 \%$ with a decrease in the concentration of $\mathrm{Ho}^{3+}$ ions from 0.5 to 2 at. $\%$.

\subsubsection{Studies of Laser Properties}

The laser properties were analyzed on the same Konoshima Chemical Co., Ltd (Kagawa, Japan). Samples [119]. The solid curves in Figure 13 depict the temperature dependence of the wavelength with maximum emission cross section $\lambda_{\max }$ in $\mathrm{Yb}_{\mathrm{Y}} \mathrm{Y}_{2} \mathrm{O}_{3}$ (red curves), $\mathrm{Yb}: \mathrm{Lu}_{2} \mathrm{O}_{3}$ (green curves) and $\mathrm{Yb}: \mathrm{Sc}_{2} \mathrm{O}_{3}$ (blue curves) ceramics samples, and in a $\mathrm{Yb}$ :YAG crystal (black curves) for comparison. The dashed curves of the corresponding color denote the values of the wavelengths at which the emission cross section is equal to half the maximal value.

The results obtained show that $\mathrm{Yb}: \mathrm{Y}_{2} \mathrm{O}_{3}$ and $\mathrm{Yb}: \mathrm{Lu}_{2} \mathrm{O}_{3}$ had almost the same central gain wavelength $\lambda_{\max }$ close to $\mathrm{Yb}$ :YAG throughout the considered temperature range. In contrast to $\mathrm{Yb}: \mathrm{YAG}$, its small shift on cooling did not play a significant role due to the wide spectrum. Actually, $\mathrm{Yb}: \mathrm{Y}_{2} \mathrm{O}_{3}$ and $\mathrm{Yb}: \mathrm{Lu}_{2} \mathrm{O}_{3}$ are not inferior to $\mathrm{Yb}: \mathrm{YAG}$, but they allow amplifying wider bandwidth pulses at an arbitrary given temperature.

Most interesting is that the central gain wavelength $\lambda_{\max }$ of Yb:YAG at $300 \mathrm{~K}$ is very close to the central gain wavelength of $\mathrm{Yb}_{\mathrm{Y}} \mathrm{Y}_{2} \mathrm{O}_{3}$ and $\mathrm{Yb}: \mathrm{Lu}_{2} \mathrm{O}_{3}$ at $80 \mathrm{~K}$. Thus, the signal of the master oscillator based on Yb:YAG crystals can be effectively amplified at the terminal, high-power cryogenic stages based on ceramic AEs made of $\mathrm{Yb}_{\mathrm{V}} \mathrm{Y}_{2} \mathrm{O}_{3}$ and $\mathrm{Yb}: \mathrm{Lu}_{2} \mathrm{O}$ ceramics. On the one hand, such a hybrid laser system, in comparison with a similar one made entirely on ceramic AEs, will reduce costs on the master oscillator, since today high optical quality laser ceramics is much more expensive than single crystals. On the other hand, shorter pulses can be obtained at the output of such a hybrid system than in a cryogenic $\mathrm{Yb}$ :YAG laser, avoiding the problem of changing the central gain wavelength on cooling. As noted above, in $\mathrm{Yb}: \mathrm{Sc}_{2} \mathrm{O}_{3}$ ceramics the central wavelength is shifted to 
longer wavelengths relative to $1030 \mathrm{~nm}$; therefore, there is currently no practical interest in using this medium.

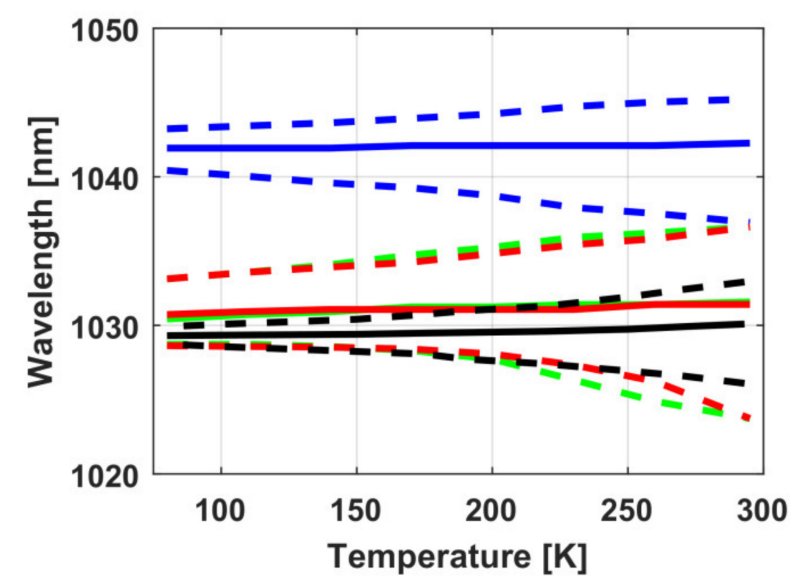

Figure 13. The wavelength with maximal emission cross section $\lambda_{\max }$ (solid curves) and wavelengths at which emission cross section is $\frac{1}{2}$ of the maximal value (dashed curves) as a function of temperature in $\mathrm{Yb}$ :YAG crystal (black curves) and in $\mathrm{Yb}: \mathrm{Y}_{2} \mathrm{O}_{3}$ (red curves), $\mathrm{Yb}_{2}: \mathrm{Lu}_{2} \mathrm{O}_{3}$ (green curves) and $\mathrm{Yb}_{2} \mathrm{Sc}_{2} \mathrm{O}_{3}$ (blue curves) ceramics samples.

The first multipass disk pulse-periodic amplifiers based on AEs of $\mathrm{Yb}: \mathrm{Y}_{2} \mathrm{O}_{3}$ ceramics operating at room as well as at cryogenic temperature were developed at the IAP RAS [120]. Their important feature is the use of the driving signal from preamplifiers on AE made of $\mathrm{Yb}: Y A G$. A disk AE made of $\mathrm{Yb}: \mathrm{Y}_{2} \mathrm{O}_{3}$ with a diameter of $15 \mathrm{~mm}$ and a thickness of $1 \mathrm{~mm}$, doped with 3.5 at.\% ytterbium ions was fabricated at Konoshima Chemical Co., Ltd.(Kagawa, Japan). The measurements of the transmission spectrum of the ceramics far from the resonance absorption peaks showed a high quality of the material (losses coincided to a high accuracy with the Fresnel reflection from two faces for a refractive index of $n=1.89$ [119], which indicates that there is no absorption or scattering in the sample). The precision measurements [121] demonstrated that the nonresonant absorption at a wavelength of $\sim 1 \mu \mathrm{m}$ is low, $\sim 0.011 / \mathrm{cm}$. The ceramic sample was placed in a vacuum cryogenic chamber with a liquid nitrogen cooling system. Since the reabsorption from the lower operating level at $77 \mathrm{~K}$ can be neglected, the lifetime at the upper laser level can be easily measured from the fluorescence decay over time. The lifetime was $1.12 \mathrm{~ms}$, which is in a good agreement with Ref. [89]. The value of the emission cross section at a wavelength of $1030 \mathrm{~nm}$ of about $1.3 \times 10^{-20} \mathrm{~cm}^{2}$ was also in a good agreement with $[89,119,122]$. As expected, upon cooling to $77 \mathrm{~K}$, the gain bandwidth at $1030 \mathrm{~m}$ became narrower, and the peak value increased. As a result, multipass disk pulse-periodic amplifiers based on $\mathrm{Yb}: \mathrm{Y}_{2} \mathrm{O}_{3}$ ceramics AEs were developed. On cooling with liquid nitrogen, the output power was $15.8 \mathrm{~W}$ at a repetition rate of $11.5 \mathrm{kHz}$, a pulse duration of $0.5 \mathrm{~ns}$, and a spectrum width of $1.2 \mathrm{~nm}$. In the case of water cooling, the output energy was $45 \mathrm{~mJ}$ at a pulse repetition rate of $10 \mathrm{~Hz}$, a pulse duration of $0.7 \mathrm{~ns}$, and a spectrum width of $2.1 \mathrm{~nm}$. According to the results of Ref. [120], the central gain wavelength of Yb:YAG at $300 \mathrm{~K}$ was very close

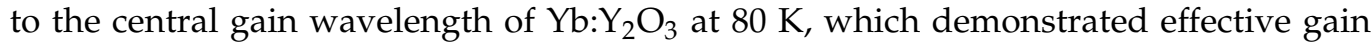
of radiation from a $\mathrm{Yb}: \mathrm{YAG}$ laser operating at room temperature in a cryogenic $\mathrm{Yb}_{\mathrm{b}} \mathrm{Y}_{2} \mathrm{O}_{3}$ amplifier without loss in spectrum width and the need to match the frequencies of cascades operating at room and cryogenic temperatures. The performed research demonstrated the high potential of using $\mathrm{Yb}: \mathrm{Y}_{2} \mathrm{O}_{3}$ ceramics for further scaling and constructing lasers with high peak power and high pulse energy. Increasing the average power by increasing the repetition rate using cryogenic cooling holds great promise. 


\subsection{Yb:LuAG Ceramics}

An increase in the concentration of the active ion for most materials leads to a decrease in thermal conductivity due to the difference in the atomic masses of the active ion and the ion that is replaced in the material, and the resulting enhancement of thermal effects. However, in $\mathrm{Yb}^{3+}$ doped materials containing $\mathrm{Lu}$, this effect of changing thermal conductivity is relatively insignificant. Lutetium aluminum garnet $\mathrm{Lu}_{3} \mathrm{Al}_{5} \mathrm{O}_{12}$ (LuAG) belongs to such materials. It possesses high values of thermal conductivity, absorption cross section, emission cross section, and mechanical strength. It can be both, grown as a single crystal and produced as a laser ceramics with high optical quality.

We studied samples of 3 at.\% $\mathrm{Yb}^{3+}: \mathrm{Lu}_{3} \mathrm{Al}_{5} \mathrm{O}_{12}$ ceramics (see Ref. [123]) made using a new method of nanocrystalline sintering without pressure in $\mathrm{H}_{2}$ (NC-PL SH) medium at the School of Material Science and Engineering, Shanghai University. For the investigation of the properties of the ceramics and obtain laser generation, the sample thickness was reduced to $0.3 \mathrm{~mm}$. The studied ceramic sample demonstrated a high, close to theoretical transmittance $(83.3 \%$ at $\lambda=1070 \mathrm{~nm})$, as well as the absorption spectra characteristic of the $\mathrm{Yb}^{3+}$ ion with a maximum of $\lambda=937 \mathrm{~nm}$ and luminescence with a maximum $\lambda=1030 \mathrm{~nm}$ and a half-height width of $6.5 \mathrm{~nm}$. The lifetime of the laser level was $0.927 \mathrm{~ms}$.

To obtain laser generation, broadband dielectric coatings were applied to the end surfaces of the $\mathrm{AE}$, antireflective on one side and reflective on the other side for the wavelength range of $930 \mathrm{~nm}-1040 \mathrm{~nm}$. The element was indium-soldered to a diamond heat sink cooled by a stream of water, and placed in a laser resonator. $12 \mathrm{~V}$-passes of pump radiation through the disk active element were organized in the resonator, which allowed over $88 \%$ of the radiation power to be absorbed in it. The maximum differential efficiency was observed when using an output mirror with $90 \%$ reflection. The power increase in disk lasers is known to be achieved by increasing the $\mathrm{AE}$ and pump spot diameters. We obtained generation for two pump spot diameters $(3$ and $5 \mathrm{~mm}$ ) on the AE. The differential efficiencies were $54 \%$ at pulsed pumping regardless of the spot diameter, and 41 and $39.3 \%$ at continuous pumping for 3 and $5 \mathrm{~mm}$ pump spot diameter, respectively. The decrease in the differential efficiency during the transition to continuous pumping can be attributed to the presence of scattering inhomogeneities in the sample bulk and to additional heat release. The efficiency can be made higher primarily due to a better fit between the pump spectrum with the maximum absorption spectrum for $\mathrm{Yb}^{3+}: \mathrm{LuAG}$, as well as due to a more optimal concentration of $\mathrm{Yb}^{3+}$ in the disk AE [124].

The generation of $200 \mathrm{~W}$ laser power at continuous pumping indicates the high quality of the ceramic material under study. Therefore, we believe that the NC-PL SH method used for the manufacturing of laser ceramics is highly promising and will allow in the future producing a high-quality ceramic material for $\mathrm{AE}$ for lasers with multikilowatt average power.

\subsection{Spinel}

$\mathrm{Yb}: \mathrm{MgAl}_{2} \mathrm{O}_{4}$ ceramics was made using the technique analogous to that described in Section 2.5.1. Ytterbium was added at the stage of hydrolysis of double magnesiumaluminate isopropoxide in the form of $\mathrm{Yb}\left(\mathrm{NO}_{3}\right)_{3}$ solution. The absorption and luminescence spectra of the obtained samples at a pumping wavelength of $940 \mathrm{~nm}$ were investigated, depending on the $\mathrm{Yb}$ content [75] (Figure 14). Low transmittance in highly doped samples and the peaks in the emission spectra at $1030 \mathrm{~nm}$ and $968 \mathrm{~nm}$ indicate the presence of the YbAG phase in the studied samples. Nevertheless, the $0.1 \%$ doped sample had a relatively high transmittance (more than $60 \%$ at $1 \mu \mathrm{m}$ ), its spectrum had no emission peaks at 1030 and $968 \mathrm{~nm}$ correlated with YbAG, there was an emission peak at $1009 \mathrm{~nm}$, which we associated with the absorption and emission of the ytterbium ion in the spinel matrix (also, there were two absorption peaks at 937 and $976 \mathrm{~nm}$ ). 


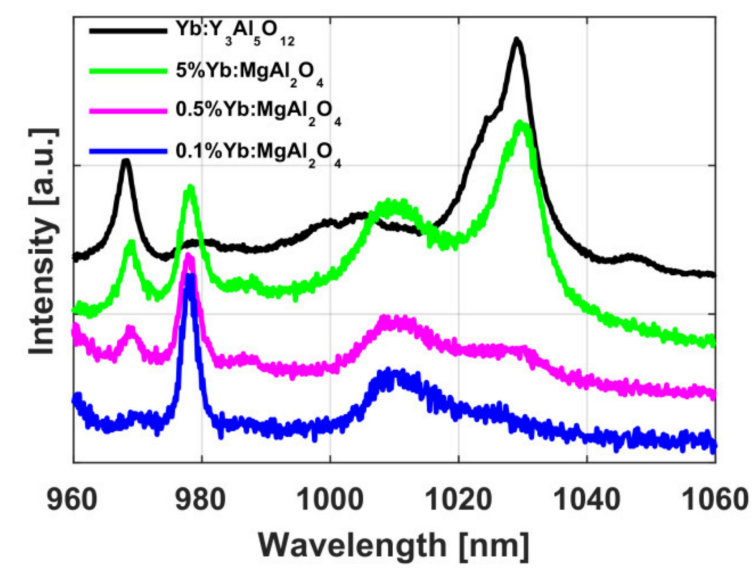

Figure 14. Luminescence spectra of MAS ceramics with the concentration of $\mathrm{Yb}^{3+}$ ions $0.1 \%$ (blue), $0.5 \%$ (magenta), and 5\% (green); black curve corresponds to the luminescence spectrum of Yb:YAG crystal.

The lower laser level was very close to the main level, which resulted in high reabsorption at the laser wavelength, but the quantum defect value was very small $(\sim 3.38 \%$ at $\lambda=976 \mathrm{~nm}$ ) due to the proximity of the pump and the laser. The upper-level lifetime in $0.1 \% \mathrm{Yb}$ ceramics was $0.7 \mathrm{~ms}$. Thus, MAS optical ceramics doped with $0.1 \% \mathrm{Yb}$ is promising for high-power applications due to its extremely low quantum defect, high thermal conductivity and mechanical properties, and possible large aperture. Therefore, we will continue to develop this trend.

\section{Conclusions}

To conclude, we would like to highlight several important points resulting from the analysis of the studies described above.

1. TGG ceramics is not inferior to TGG crystals as a medium for the FIs for highaverage-power lasers, including at nitrogen temperatures. At the same time, it allows constructing large-aperture devices, thus opening up new opportunities for developing lasers with high time-average and peak power.

2. The growth technology of TAG ceramics allows introducing into its composition both, sintering additives and additional ions, which can affect the Verdet constant. However, doping of TAG ceramics with $\mathrm{Ce}, \mathrm{Pr}, \mathrm{Ho}, \mathrm{Si}, \mathrm{Ti}$, and $\mathrm{Zr}$ in small concentrations (up to 2 at.\%) does not give a significant increase in the Verdet constant. Besides, Re:TAG ceramics, that is superior to TGG in the Verdet constant, can be successfully applied in high-power FI, after further improvement of its optical properties.

3. $\mathrm{Tb}_{2} \mathrm{O}_{3}, \mathrm{Dy}_{2} \mathrm{O}_{3}$ and $\mathrm{Ho}_{2} \mathrm{O}_{3}$ sesquioxide ceramics, which are also superior to TGG in the Verdet constant, are potentially interesting throughout their transparency ranges/windows. Within the range up to $\sim 1.4 \mu \mathrm{m}$, the most preferable materials are $\mathrm{Tb}_{2} \mathrm{O}_{3}$ and TAG (partly $\mathrm{Dy}_{2} \mathrm{O}_{3}$ ); in the range of 1.5-2.1 $\mu \mathrm{m}, \mathrm{Dy}_{2} \mathrm{O}_{3}$ or $\mathrm{Ho}_{2} \mathrm{O}_{3}$ (at lower or higher $\lambda$, respectively); and in the 2.1-3.2 $\mu \mathrm{m}$ range, $\mathrm{Ho}_{2} \mathrm{O}_{3}$ is a leader; in the $\sim 3.2-6 \mu \mathrm{m}$ range, $\mathrm{Dy}_{2} \mathrm{O}_{3}$ is a leader; and in the range of $6-8 \mu \mathrm{m}, \mathrm{Tb}_{2} \mathrm{O}_{3}$ returns its leadership. Being paramagnetic, these ceramics will be of even greater interest in cryogenically cooled devices after further improvement of their optical properties.

4. Polycrystalline zinc chalcogenides ( $\mathrm{ZnSe}, \mathrm{ZnS}$ diamagnets) are promising MAEs for high-power FIs in their transparency windows. In particular, a high-purity CVD-ZnSe can be used for developing an FI at $\lambda=1 \mu \mathrm{m}$ with a multikilowatt $P_{\text {max }}$ operating at room temperature.

5. The magneto-optical characteristics of magnesium-aluminate and zinc-aluminate spinels are significantly (more than an order of magnitude) inferior to the popular (above mentioned) MAEs. Doping with $\mathrm{Sc}, \mathrm{Ti}, \mathrm{Tb}, \mathrm{Ce}, \mathrm{Zr}$ and $\mathrm{Yb}$ ions in small concentrations ( 1 at.\%), like in TAG, does not lead to a significant increase in the 
Verdet constant. All samples demonstrate the diamagnetic rotation of the polarization plane.

6. The developed disk cryogenic laser based on Yb:YAG ceramics AE gives us ground to state that the quality of $Y b$ :YAG ceramics is not inferior to that of Yb:YAG crystal. The achieved value of $233 \mathrm{~mJ}$ in 70 -ns pulses at a repetition rate of $143 \mathrm{~Hz}$ with a high $(20 \%)$ efficiency is the best among cryogenic pulse-periodic laser systems based on thin Yb:YAG ceramic disks developed at the time of this publication.

7. The analysis of the luminescence spectrum in the visible range of $\mathrm{AE}$ made of sesquioxide ceramics (for example, $\mathrm{Yb}: \mathrm{Y}_{2} \mathrm{O}_{3}$ ceramics) shows the laser quality of ceramics in terms of chemical purity.

8. The comparison of the popular $\mathrm{Y}_{2} \mathrm{O}_{3}, \mathrm{Lu}_{2} \mathrm{O}_{3}$, and $\mathrm{Sc}_{2} \mathrm{O}_{3}$ sesquioxide ceramics doped with a $\mathrm{Yb}^{3+}$ ion showed that the $\mathrm{Lu}_{2} \mathrm{O}_{3}$ and $\mathrm{Sc}_{2} \mathrm{O}_{3}$ materials have similar values of thermo-optical constants $Q_{e f f}$ that is $30 \%$ less in $\mathrm{Y}_{2} \mathrm{O}_{3}$. Therefore, the thermally induced depolarization in the latter will be $\sim 2$ times less. To fabricate AEs with a high (>4 at.\%) concentration of $\mathrm{Yb}^{3+}$ ions, from the point of view of minimizing thermally induced polarization distortions, it is more advantageous to use $\mathrm{Lu}_{2} \mathrm{O}_{3}$ ceramics. Contrariwise, for AEs with a lower $\mathrm{Yb}^{3+}$ concentration, it is more reasonable to use $\mathrm{Sc}_{2} \mathrm{O}_{3}$ ceramics. The comparison of the thermo-optical constant $P$ singles out the $\mathrm{Sc}_{2} \mathrm{O}_{3}$ material, in which this value is significantly lower than in $\mathrm{Y}_{2} \mathrm{O}_{3}$ and $\mathrm{Lu}_{2} \mathrm{O}_{3}$. (The lower $P$, the lower the optical power of the thermal lens introduced by the $\mathrm{AE}$ bulk is).

9. The $\mathrm{Yb}: \mathrm{Y}_{2} \mathrm{O}_{3}$ and $\mathrm{Yb}: \mathrm{Lu}_{2} \mathrm{O}_{3}$ ceramics are in no way inferior to the $\mathrm{Yb}: \mathrm{YAG}$ material, but they allow amplifying wider-bandwidth pulses at any temperature. Most interesting is the fact that the central gain wavelength $\lambda_{\max }$ of $\mathrm{Yb}: \mathrm{YAG}$ at $300 \mathrm{~K}$ is very close to the central gain wavelength of $\mathrm{Yb}: \mathrm{Y}_{2} \mathrm{O}_{3}$ and $\mathrm{Yb}: \mathrm{Lu}_{2} \mathrm{O}_{3}$ at $80 \mathrm{~K}$. Thus, the signal of the master oscillator based on Yb:YAG crystals can be effectively amplified at the powerful terminal cryogenic amplification stages based on ceramic AEs made of $\mathrm{Yb}: \mathrm{Y}_{2} \mathrm{O}_{3}$ and $\mathrm{Yb}: \mathrm{Lu}_{2} \mathrm{O}_{3}$. In $\mathrm{Yb}: \mathrm{Sc}_{2} \mathrm{O}_{3}$ ceramics, the central wavelength is shifted to a longer wavelength region therefore, in our opinion, there is no practical interest in its use at the present.

10. Despite the obvious advantages, and in some cases the irreplaceability of rare-earth oxide ceramics, their wide use in high-power laser engineering is currently limited by the complexity and weak reproducibility of their technologies. This ceramics is currently just at the beginning of its development. But there is no doubt that like the advance of the CVD-ZnSe technologies in the 1980-90 s or of the YAG ceramics at the beginning of this century, there will be progress in the production of rare-earth oxide ceramics. The scientific research and the competition will lead to a significant reduction in the cost of these materials, to improved quality and, eventually, to their widespread commercial use, hopefully in the nearest future.

Author Contributions: Conceptualization, O.V.P., A.V.S., and S.S.B.; methodology, O.V.P.; validation, A.V.S., E.A.P., I.L.S., E.A.M., A.I.Y., D.A.P. and A.V.B.; formal analysis, A.V.S., E.A.P., I.L.S., E.A.M., A.I.Y., S.S.B., D.A.P., A.V.B.; investigation, A.V.S., E.A.P., I.L.S., E.A.M., A.I.Y., S.S.B., D.A.P. and A.V.B.; writing-original draft preparation, O.V.P., A.V.S., E.A.P., I.L.S., E.A.M., and S.S.B.; writing-review and editing, O.V.P. and A.V.S.; visualization, A.V.S.; supervision, O.V.P. and S.S.B.; funding acquisition, O.V.P. and S.S.B. All authors have read and agreed to the published version of the manuscript.

Funding: Magneto-active sesquioxide ceramics fabrication was supported by the Russian Science Foundation [No. 18-13-00355], https:/ / rscf.ru/en/project/18-13-00355/ (accessed on 13 July 2021). Analysis of the properties of ceramics in order to use them in lasers with simultaneously high peak and time-average power was supported by the Russian Science Foundation [No. 18-12-00416], https:/ / rscf.ru/en/project/18-12-00416/ (accessed on 13 July 2021).

Institutional Review Board Statement: Not applicable.

Informed Consent Statement: Not applicable. 
Data Availability Statement: The authors confirm that the data supporting the findings of this study are available at the references given in the text of the article and accordingly listed in the reference list. The analyzed data are available from the corresponding author, O.V.P., upon reasonable request.

Conflicts of Interest: The authors declare no conflict of interest. The funders had no role in the design of the study; in the collection, analyses, or interpretation of data; in the writing of the manuscript, or in the decision to publish the results.

\section{References}

1. Khazanov, E.A. Thermally induced birefringence in Nd:YAG ceramics. Opt. Lett. 2002, 27, 716. [CrossRef]

2. Kagan, M.A.; Khazanov, E.A. Thermally induced birefringence in Faraday devices made from terbium gallium garnetpolycrystalline ceramics. Appl. Opt. 2004, 43, 6030-6039. [CrossRef]

3. Kagan, M.A.; Khazanov, E.A. Compensation for thermally induced birefringence in polycrystalline ceramic active elements. Quantum Electron. 2003, 33, 876-882. [CrossRef]

4. Mukhin, I.B.; Palashov, O.V.; Khazanov, E.A.; Ikesue, A.; Aung, Y.L. Experimental study of thermally induced depolarization in Nd:YAG ceramics. Opt. Express 2005, 13, 5983. [CrossRef]

5. Soloviev, A.; Snetkov, I.; Zelenogorsky, V.; Kozhevatov, I.; Palashov, O.; Khazanov, E. Experimental study of thermal lens features in laser ceramics. Opt. Express 2008, 16, 21012. [CrossRef] [PubMed]

6. Palashov, O.V.; Khazanov, E.A.; Mukhin, I.B.; Smirnov, A.N.; Mironov, I.A.; Dukel'skii, K.V.; Garibin, E.A.; Fedorov, P.P.; Kuznetsov, S.V.; Osiko, V.V.; et al. Optical absorption in $\mathrm{CaF}_{2}$ nanoceramics. Quantum Electron. 2009, 39, 943-947. [CrossRef]

7. Zheleznov, D.S.; Voitovich, A.V.; Mukhin, I.B.; Palashov, O.V.; Khazanov, E.A. Considerable reduction of thermooptical distortions in Faraday isolators cooled to 77 K. Quantum Electron. 2006, 36, 383-388. [CrossRef]

8. Mezenov, A.V.; Soms, L.N.; Stepanov, A.I. Thermooptics of Solid-State Lasers; Mashinebuilding: Leningrad, Russia, 1986.

9. Khazanov, E.A. Thermooptics of magnetoactive medium: Faraday isolators for high average power lasers. Uspekhi Fiz. Nauk 2016, 186, 975-1000. [CrossRef]

10. Khazanov, E. Faraday Isolators for High Average Power Lasers. In Advances in Solid State Lasers Development and Applications; InTech: Nizhny Novgorod, Russia, 2010; Volume 13, pp. 45-72. ISBN 9789537619800.

11. Vyatkin, A.G.; Khazanov, E.A. Thermally induced scattering of radiation in laser ceramics with arbitrary grain size. J. Opt. Soc. Am. B 2012, 29, 3307. [CrossRef]

12. Mironov, E.A.; Voitovich, A.V.; Starobor, A.V.; Palashov, O.V. Compensation of polarization distortions in Faraday isolators by means of magnetic field inhomogeneity. Appl. Opt. 2014, 53, 3486. [CrossRef]

13. Khazanov, E.A.; Kulagin, O.V.; Yoshida, S.; Tanner, D.B.; Reitze, D.H. Investigation of self-induced depolarization of laser radiation in terbium gallium garnet. IEEE J. Quantum Electron. 1999, 35, 1116-1122. [CrossRef]

14. Starobor, A.V.; Zheleznov, D.S.; Palashov, O.V.; Khazanov, E.A. Magnetoactive media for cryogenic Faraday isolators. J. Opt. Soc. Am. B 2011, 28, 1409. [CrossRef]

15. Zheleznov, D.S.; Starobor, A.V.; Palashov, O.V.; Khazanov, E.A. Cryogenic Faraday isolator with a disk-shaped magneto-optical element. J. Opt. Soc. Am. B 2012, 29, 786. [CrossRef]

16. Mironov, E.A.; Voitovich, A.V.; Starobor, A.V.; Zheleznov, D.S.; Palashov, O.V. Effect of paramagnetic magnetization in Faraday isolators. Appl. Opt. 2012, 51, 5073. [CrossRef]

17. Slezak, O.; Yasuhara, R.; Lucianetti, A.; Mocek, T. Wavelength dependence of magneto-optic properties of terbium gallium garnet ceramics. Opt. Express 2015, 23, 13641. [CrossRef]

18. Yasuhara, R.; Tokita, S.; Kawanaka, J.; Kan, H.; Kawashima, T.; Yagi, H.; Nozawa, H.; Yanagitani, T.; Fugimoto, Y.; Yoshida, H.; et al. Novel Faraday Rotator by Use of Cryogenic TGG Ceramics. Rev. Laser Eng. 2008, 36, 1306-1309. [CrossRef]

19. Yasuhara, R.; Furuse, H. Thermally induced depolarization in TGG ceramics. Opt. Lett. 2013, 38, 1751. [CrossRef] [PubMed]

20. Yasuhara, R.; Snetkov, I.; Starobor, A.V.; Zheleznov, D.; Palashov, O.; Khazanov, E.; Nozawa, H.; Yanagitani, T. Terbium gallium garnet ceramic Faraday rotator for high-power laser application. Opt. Lett. 2014, 39, 1145. [CrossRef] [PubMed]

21. Snetkov, I.L.; Yasuhara, R.; Starobor, A.V.; Palashov, O.V. TGG ceramics based Faraday isolator with external compensation of thermally induced depolarization. Opt. Express 2014, 22, 4144. [CrossRef]

22. Yasuhara, R.; Snetkov, I.; Starobor, A.; Palashov, O. Terbium gallium garnet ceramic-based Faraday isolator with compensation of thermally induced depolarization for high-energy pulsed lasers with kilowatt average power. Appl. Phys. Lett. 2014, 105, 241104. [CrossRef]

23. Starobor, A.V.; Yasuhara, R.; Zheleznov, D.S.; Palashov, O.V.; Khazanov, E.A. Cryogenic Faraday Isolator Based on TGG Ceramics. IEEE J. Quantum Electron. 2014, 50, 749-754. [CrossRef]

24. Mironov, E.A.; Snetkov, I.L.; Voitovich, A.V.; Palashov, O.V. Permanent-magnet Faraday isolator with the field intensity of 25 kOe. Quantum Electron. 2013, 43, 740-743. [CrossRef]

25. Starobor, A.V.; Palashov, O.; Zhou, S. Thermo-optical properties of terbium-aluminum garnet ceramics doped with silicon and titanium. Opt. Lett. 2016, 41, 1510. [CrossRef] [PubMed]

26. Khazanov, E.A. Compensation of thermally induced polarisation distortions in Faraday isolators. Quantum Electron. 1999, 29, 59-64. [CrossRef] 
27. Snetkov, I.; Mukhin, I.; Palashov, O.; Khazanov, E. Compensation of thermally induced depolarization in Faraday isolators for high average power lasers. Opt. Express 2011, 19, 6366. [CrossRef] [PubMed]

28. Rubinstein, C.B.; Van Uitert, L.G.; Grodkiewicz, W.H. Magneto-optical properties of rare earth (III) aluminum garnets. J. Appl. Phys. 1964, 35, 3069-3070. [CrossRef]

29. Geho, M.; Takagi, T.; Chiku, S.; Fujii, T. Development of Optical Isolators for Visible Light Using Terbium Aluminum Garnet (Tb3A15O12 ) Single Crystals. Jpn. J. Appl. Phys. 2005, 44, 4967-4970. [CrossRef]

30. Geho, M.; Sekijima, T.; Fujii, T. Growth of terbium aluminum garnet $\left(\mathrm{Tb}_{3} \mathrm{Al}_{5} \mathrm{O}_{12} ; \mathrm{TAG}\right)$ single crystals by the hybrid laser floating zone machine. J. Cryst. Growth 2004, 267, 188-193. [CrossRef]

31. Ganschow, S.; Klimm, D.; Reiche, P.; Uecker, R. On the Crystallization of Terbium Aluminium Garnet. Cryst. Res. Technol. 1999, 34, 615-619. [CrossRef]

32. Ganschow, S.; Klimm, D.; Epelbaum, B.M.; Yoshikawa, A.; Doerschel, J.; Fukuda, T. Growth conditions and composition of terbium aluminum garnet single crystals grown by the micro pulling down technique. J. Cryst. Growth 2001, 225, $454-457$. [CrossRef]

33. Lin, H.; Zhou, S.; Teng, H. Synthesis of $\mathrm{Tb}_{3} \mathrm{Al}_{5} \mathrm{O}_{12}$ (TAG) transparent ceramics for potential magneto-optical applications. Opt. Mater. 2011, 33, 1833-1836. [CrossRef]

34. Hao, D.; Feng, Y.; Tang, Y.; Yi, X.; Zhang, S.; Chen, C.; Lin, H.; Zhou, S. $\mathrm{Tb}_{3} \mathrm{Al}_{2.5} \mathrm{Ga}_{2.5} \mathrm{O}_{12}$ Transparent Ceramic for Magneto-Optical Application. Int. J. Appl. Ceram. Technol. 2016, 13, 816-820. [CrossRef]

35. Dai, J.; Snetkov, I.L.; Palashov, O.V.; Pan, Y.; Kou, H.; Li, J. Fabrication, microstructure and magneto-optical properties of $\mathrm{Tb}_{3} \mathrm{Al}_{5} \mathrm{O}_{12}$ transparent ceramics. Opt. Mater. 2016, 62, 205-210. [CrossRef]

36. Hamamoto, K.; Nishio, M.; Tokita, S.; Uehara, H.; Yanagitani, T.; Fujioka, K.; Yasuhara, R.; Kawanaka, J. Properties of TAG ceramics at room and cryogenic temperatures and performance estimations as a Faraday isolator. Opt. Mater. Express 2021, 11, 434. [CrossRef]

37. Chen, C.; Ni, Y.; Zhou, S.; Lin, H.; Yi, X. Preparation of $\left(\mathrm{Tb}_{0.8} \mathrm{Y}_{0.2}\right)_{3} \mathrm{Al}_{5} \mathrm{O}_{12}$ transparent ceramic as novel magneto-optical isolator material. Chin. Opt. Lett. 2013, 11, 21601-21603. [CrossRef]

38. Yakovlev, A.I.; Snetkov, I.L.; Palashov, O.V.; Dai, J.; Li, X.; Li, J. Magneto-Optical and Thermo-Optical Properties of Ce, Pr, and Ho Doped TAG Ceramics. IEEE J. Quantum Electron. 2019, 55, 7000108. [CrossRef]

39. Snetkov, I.L.; Permin, D.A.; Balabanov, S.S.; Palashov, O. V Wavelength dependence of Verdet constant of $\mathrm{Tb}^{3+}: \mathrm{Y}_{2} \mathrm{O}_{3}$ ceramics. Appl. Phys. Lett. 2016, 108, 161905. [CrossRef]

40. Balabanov, S.S.; Permin, D.A.; Rostokina, E.Y.; Palashov, O.V.; Snetkov, I.L. Characterizations of REE:Tb $\mathrm{O}_{3} \mathrm{Magneto} \mathrm{Optical}$ Ceramics. Phys. Status Solidi B 2020, 257, 1900474. [CrossRef]

41. Starobor, A.V.; Zheleznov, D.; Palashov, O.; Chen, C.; Zhou, S.; Yasuhara, R. Study of the properties and prospects of Ce:TAG and TGG magnetooptical ceramics for optical isolators for lasers with high average power. Opt. Mater. Express 2014, 4, 2127. [CrossRef]

42. Snetkov, I.L.; Yakovlev, A.I.; Permin, D.A.; Balabanov, S.S.; Palashov, O.V. Magneto-optical Faraday effect in dysprosium oxide $\left(\mathrm{Dy}_{2} \mathrm{O}_{3}\right)$ based ceramics obtained by vacuum sintering. Opt. Lett. 2018, 43, 4041. [CrossRef]

43. Balabanov, S.; Filofeev, S.; Ivanov, M.; Kaigorodov, A.; Kuznetsov, D.; Hu, D.J.; Li, J.; Palashov, O.; Permin, D.; Rostokina, E.; et al. Fabrication and characterizations of holmium oxide based magneto-optical ceramics. Opt. Mater. 2020, 101, 109741. [CrossRef]

44. Hu, D.; Li, X.; Snetkov, I.; Yakovlev, A.; Balabanov, S.S.; Ivanov, M.; Liu, X.; Liu, Z.; Tian, F.; Xie, T.; et al. Fabrication, microstructure and optical characterizations of holmium oxide $\left(\mathrm{Ho}_{2} \mathrm{O}_{3}\right)$ transparent ceramics. J. Eur. Ceram. Soc. 2021, 41, 759-767. [CrossRef]

45. Balabanov, S.; Filofeev, S.; Ivanov, M.; Kaigorodov, A.; Krugovykh, A.; Kuznetsov, D.; Permin, D.; Popov, P.; Rostokina, E. Fabrication and characterizations of erbium oxide based optical ceramics. Opt. Mater. 2020, 101, 109732. [CrossRef]

46. Permin, D.A.; Novikova, A.V.; Koshkin, V.A.; Balabanov, S.S.; Snetkov, I.L.; Palashov, O.V.; Smetanina, K.E. Fabrication and Magneto-Optical Properties of $\mathrm{Yb}_{2} \mathrm{O}_{3}$ Based Ceramics. Magnetochemistry 2020, 6, 63. [CrossRef]

47. Mironov, E.A.; Palashov, O.V.; Snetkov, I.L.; Balabanov, S.S. ZnSe-based Faraday isolator for high-power mid-IR lasers. Laser Phys. Lett. 2020, 17, 125801. [CrossRef]

48. Zheleznov, D.; Starobor, A.V.; Palashov, O.; Chen, C.; Zhou, S. High-power Faraday isolators based on TAG ceramics. Opt. Express 2014, 22, 2578-2583. [CrossRef] [PubMed]

49. Zheleznov, D.; Starobor, A.V.; Palashov, O.; Lin, H.; Zhou, S. Improving characteristics of Faraday isolators based on TAG ceramics by cerium doping. Opt. Lett. 2014, 39, 2183-2186. [CrossRef]

50. Chen, J.; Lin, H.; Hao, D.; Tang, Y.; Yi, X.; Zhao, Y.; Zhou, S. Exaggerated grain growth caused by $\mathrm{ZrO}_{2}$-doping and its effect on the optical properties of $\mathrm{Tb}_{3} \mathrm{Al}_{5} \mathrm{O}_{12}$ ceramics. Scr. Mater. 2019, 162, 82-85. [CrossRef]

51. Starobor, A.V.; Zheleznov, D.S.S.; Palashov, O.V.V. The temperature dependence of the thermo-optical properties of TAG optical ceramics. Laser Phys. 2016, 26, 025801. [CrossRef]

52. Starobor, A.V.; Palashov, O. The temperature dependence of thermooptical properties of magnetooptical TAG ceramics doped with silicon and titanium. Opt. Mater. 2018, 78, 15-20. [CrossRef]

53. Snetkov, I.L.; Palashov, O.V. Cryogenic temperature characteristics of Verdet constant of terbium sesquioxide ceramics. Opt. Mater. 2016, 62, 697-700. [CrossRef]

54. Yakovlev, A.; Snetkov, I.; Permin, D.; Balabanov, S.S.; Palashov, O. Faraday rotation in cryogenically cooled dysprosium based $\left(\mathrm{Dy}_{2} \mathrm{O}_{3}\right)$ ceramics. Scr. Mater. 2019, 161, 32-35. [CrossRef] 
55. Klein, P.H.; Croft, W.J. Thermal Conductivity, Diffusivity, and Expansion of $\mathrm{Y}_{2} \mathrm{O}_{3}, \mathrm{Y}_{3} \mathrm{Al}_{5} \mathrm{O}_{12}$, and $\mathrm{LaF}_{3}$ in the Range $77^{\circ}-300^{\circ} \mathrm{K}$. J. Appl. Phys. 1967, 38, 1603-1607. [CrossRef]

56. Zhou, D.; Li, X.; Wang, T.; Xu, J.; Wang, Z.; Shi, Y.; Permin, D.; Balabanov, S.S. Fabrication and Magneto-Optical Property of $\left(\mathrm{Dy}_{0.7} \mathrm{Y}_{0.25} \mathrm{La}_{0.05}\right)_{2} \mathrm{O}_{3}$ Transparent Ceramics by PLSH Technology. Magnetochemistry 2020, 6, 70. [CrossRef]

57. Yakovlev, A.; Balabanov, S.S.; Permin, D.; Ivanov, M.; Snetkov, I. Faraday rotation in erbium oxide based ceramics. Opt. Mater. 2020, 101, 109750. [CrossRef]

58. Balabanov, S.S.; Filofeev, S.V.; Ivanov, M.G.; Kalinina, E.G.; Kuznetsov, D.K.; Permin, D.A.; Rostokina, E.Y. Self-propagating high-temperature synthesis of $\left(\mathrm{Ho}_{1-x} \mathrm{La}_{x}\right)_{2} \mathrm{O}_{3}$ nanopowders for magneto-optical ceramics. Heliyon 2019, 5, e01519. [CrossRef]

59. Balabanov, S.S.; Permin, D.A.; Rostokina, E.Y.; Egorov, S.V.; Sorokin, A.A. Sinterability of nanopowders of terbia solid solutions with scandia, yttria, and lutetia. J. Adv. Ceram. 2018, 7, 362-369. [CrossRef]

60. Balabanov, S.S.; Permin, D.A.; Rostokina, E.Y.; Egorov, S.V.; Sorokin, A.A.; Kuznetsov, D.D. Synthesis and structural characterization of ultrafine terbium oxide powders. Ceram. Int. 2017, 43, 16569-16574. [CrossRef]

61. Balabanov, S.; Demidova, K.; Filofeev, S.; Ivanov, M.; Kuznetsov, D.; Li, J.; Permin, D.; Rostokina, E. Influence of Lanthanum Concentration on Microstructure of $\left(\mathrm{Ho}_{1-x} \mathrm{La}_{x}\right)_{2} \mathrm{O}_{3}$ Magneto-Optical Ceramics. Phys. Status Solidi B 2020, 257, 1900500. [CrossRef]

62. Slezák, O.; Yasuhara, R.; Vojna, D.; Furuse, H.; Lucianetti, A.; Mocek, T. Temperature-wavelength dependence of Verdet constant of $\mathrm{Dy}_{2} \mathrm{O}_{3}$ ceramics. Opt. Mater. Express 2019, 9, 2971. [CrossRef]

63. Snetkov, I.L.; Yasuhara, R.; Starobor, A.V.; Mironov, E.A.; Palashov, O.V. Thermo-Optical and Magneto-Optical Characteristics of Terbium Scandium Aluminum Garnet Crystals. IEEE J. Quantum Electron. 2015, 51, 7000307. [CrossRef]

64. Van Vleck, J.H.; Hebb, M.H. On the Paramagnetic Rotation of Tysonite. Phys. Rev. 1934, 46, 17-32. [CrossRef]

65. Gavrushchuk, E.M. Polycrystalline Zinc Selenide for IR Optical Applications. Inorg. Mater. 2003, 39, 883-899. [CrossRef]

66. Mironov, E.A.; Palashov, O.V.; Balabanov, S.S. High-purity CVD-ZnSe polycrystal as a magneto-active medium for a multikilowatt Faraday isolator. Opt. Lett. 2021, 46, 2119-2122. [CrossRef]

67. Balabanov, S.S.; Belyaev, A.V.; Popov, P.A. Heat Conduction of Ceramic Materials Based on $\mathrm{MgAl}_{2} \mathrm{O}_{4}$ and $\mathrm{ZnAl}_{2} \mathrm{O}_{4}$. J. Eng. Phys. Thermophys. 2020, 93, 719-724. [CrossRef]

68. Alekseev, M.K.; Kulikova, G.I.; Rusin, M.Y.; Savanina, N.N.; Balabanov, S.S.; Belyaev, A.V.; Gavrishchuk, E.M.; Ivanov, A.V.; Rizakhanov, R.N. Transparent ceramics prepared from ultrapure magnesium aluminate spinel nanopowders by spark plasma sintering. Inorg. Mater. 2016, 52, 324-330. [CrossRef]

69. Belyaev, A.V.; Lelet, M.I.; Kirillova, N.I.; Khamaletdinova, N.M.; Boldin, M.S.; Murashov, A.A.; Balabanov, S.S. Sol-gel synthesis and characterization of $\mathrm{ZnAl}_{2} \mathrm{O}_{4}$ powders for transparent ceramics. Ceram. Int. 2019, 45, 4835-4839. [CrossRef]

70. Balabanov, S.S.; Vaganov, V.E.; Gavrishchuk, E.M.; Drobotenko, V.V.; Permin, D.A.; Fedin, A.V. Effect of magnesium aluminum isopropoxide hydrolysis conditions on the properties of magnesium aluminate spinel powders. Inorg. Mater. 2014, 50, 830-836. [CrossRef]

71. Balabanov, S.S.; Yavetskiy, R.P.; Belyaev, A.V.; Gavrishchuk, E.M.; Drobotenko, V.V.; Evdokimov, I.I.; Novikova, A.V.; Palashov, O.V.; Permin, D.A.; Pimenov, V.G. Fabrication of transparent $\mathrm{MgAl}_{2} \mathrm{O}_{4}$ ceramics by hot-pressing of sol-gel-derived nanopowders. Ceram. Int. 2015, 41, 13366-13371. [CrossRef]

72. Waetzig, K.; Hutzler, T. Highest UV-vis transparency of $\mathrm{MgAl}_{2} \mathrm{O}_{4}$ spinel ceramics prepared by hot pressing with LiF. J. Eur. Ceram. Soc. 2017, 37, 2259-2263. [CrossRef]

73. Belyaev, A.; Basyrova, L.; Sysoev, V.; Lelet, M.; Balabanov, S.S.; Kalganov, V.; Mikhailovski, V.; Baranov, M.; Stepanidenko, E.; Vitkin, $\mathrm{V}$; et al. Microstructure, doping and optical properties of $\mathrm{Co}^{2+}: \mathrm{ZnAl}_{2} \mathrm{O}_{4}$ transparent ceramics for saturable absorbers: Effect of the $\mathrm{ZnF}_{2}$ sintering additive. J. Alloys Compd. 2020, 829, 154514. [CrossRef]

74. Basyrova, L.; Bukina, V.; Balabanov, S.S.; Belyaev, A.; Drobotenko, V.; Dymshits, O.; Alekseeva, I.; Tsenter, M.; Zapalova, S.; Khubetsov, A.; et al. Synthesis, structure and spectroscopy of $\mathrm{Fe}^{2+}: \mathrm{MgAl}_{2} \mathrm{O}_{4}$ transparent ceramics and glass-ceramics. J. Lumin . 2021, 236, 118090. [CrossRef]

75. Balabanov, S.S.; Belyaev, A.V.; Gavrishchuk, E.M.; Mukhin, I.B.; Novikova, A.V.; Palashov, O.V.; Permin, D.A.; Snetkov, I.L. Fabrication and measurement of optical and spectral properties of the transparent $\mathrm{Yb}: \mathrm{MgAl}_{2} \mathrm{O}_{4}$ ceramics. Opt. Mater. 2017, 71, 17-22. [CrossRef]

76. Reagan, B.A.; Berrill, M.; Wernsing, K.A.; Baumgarten, C.; Woolston, M.; Rocca, J.J. High-average-power, 100-Hz-repetition-rate, tabletop soft-x-ray lasers at sub-15-nm wavelengths. Phys. Rev. A 2014, 89, 53820. [CrossRef]

77. Pupeza, I.; Sánchez, D.; Zhang, J.; Lilienfein, N.; Seidel, M.; Karpowicz, N.; Paasch-Colberg, T.; Znakovskaya, I.; Pescher, M.; Schweinberger, W.; et al. High-power sub-two-cycle mid-infrared pulses at $100 \mathrm{MHz}$ repetition rate. Nat. Photonics 2015, 9, 721-724. [CrossRef]

78. Schneider, W.; Ryabov, A.; Lombosi, C.; Metzger, T.; Major, Z.; Fülöp, J.A.; Baum, P. 800-fs, 330uJ pulses from a 100-W regenerative Yb:YAG thin-disk amplifier at $300 \mathrm{kHz}$ and $\mathrm{THz}$ generation in $\mathrm{LiNbO}_{3}$. Opt. Lett. 2014, 39, 6604-6607. [CrossRef] [PubMed]

79. Zapata, L.E.; Lin, H.; Calendron, A.-L.; Cankaya, H.; Hemmer, M.; Reichert, F.; Huang, W.R.; Granados, E.; Hong, K.-H.; Kärtner, F.X. Cryogenic Yb:YAG composite-thin-disk for high energy and average power amplifiers. Opt. Lett. 2015, 40, 2610-2613. [CrossRef]

80. Baumgarten, C.; Pedicone, M.; Bravo, H.; Wang, H.; Yin, L.; Menoni, C.S.; Rocca, J.J.; Reagan, B.A. 1 J, 0.5 kHz repetition rate picosecond laser. Opt. Lett. 2016, 41, 3339-3342. [CrossRef] 
81. HiLASE Centre Institute of Physics of the Czech Academy of Sciences. Available online: https://www.hilase.cz/en/ (accessed on 13 July 2021).

82. Danson, C.N.; Haefner, C.; Bromage, J.; Butcher, T.; Chanteloup, J.C.F.; Chowdhury, E.A.; Galvanauskas, A.; Gizzi, L.A.; Hein, J.; Hillier, D.I.; et al. Petawatt and exawatt class lasers worldwide. High Power Laser Sci. Eng. 2019, 7. [CrossRef]

83. Banerjee, S.; Ertel, K.; Mason, P.D.; Phillips, P.J.; Siebold, M.; Loeser, M.; Hernandez-Gomez, C.; Collier, J.L. High-efficiency 10 J diode pumped cryogenic gas cooled Yb:YAG multislab amplifier. Opt. Lett. 2012, 37, 2175. [CrossRef]

84. Kaksis, E.; Almási, G.; Fülöp, J.A.; Pugžlys, A.; Baltuška, A.; Andriukaitis, G. 110-mJ 225-fs cryogenically cooled Yb:CaF 2 multipass amplifier. Opt. Express 2016, 24, 28915. [CrossRef]

85. João, C.P.; Wagner, F.; Körner, J.; Hein, J.; Gottschall, T.; Limpert, J.; Bagnoud, V. A 10-mJ-level compact CPA system based on $\mathrm{Yb}$ :KGW for ultrafast optical parametric amplifier pumping. Appl. Phys. B Lasers Opt. 2015, 118, 401-407. [CrossRef]

86. Kim, G.H.; Yang, J.; Lee, B.; Kim, J.W.; Heo, D.C.; Chizhov, S.A.; Sall', E.G.; Yashin, V.E. High-power repetitively pulsed ytterbium lasers with supershort pulse width and direct diode pumping for technological and biomedical applications. J. Opt. Technol. 2018, 85, 679. [CrossRef]

87. Lu, J.; Song, J.; Prabhu, M.; Xu, J.; Ueda, K.; Yagi, H.; Yanagitani, T.; Kudryashov, A. High-Power $\mathrm{Nd}_{2} \mathrm{Y}_{3} \mathrm{Al}_{5} \mathrm{O}_{12} \mathrm{Ceramic}_{2}$ aser. Jpn. J. Appl. Phys. 2000, 39, L1048-L1050. [CrossRef]

88. Kong, J.; Tang, D.Y.; Lu, J.; Ueda, K. Spectral characteristics of a Yb-doped $\mathrm{Y}_{2} \mathrm{O}_{3}$ ceramic laser. Appl. Phys. B 2004, 79, 449-455. [CrossRef]

89. Merkle, L.D.; Alex Newburgh, G.; Ter-Gabrielyan, N.; Michael, A.; Dubinskii, M. Temperature-dependent lasing and spectroscopy of $\mathrm{Yb}: \mathrm{Y}_{2} \mathrm{O}_{3}$ and $\mathrm{Yb}: \mathrm{Sc}_{2} \mathrm{O}_{3}$. Opt. Commun. 2008, 281, 5855-5861. [CrossRef]

90. Pirri, A.; Toci, G.; Vannini, M. First laser oscillation and broad tunability of 1 at. \% Yb-doped $\mathrm{Sc}_{2} \mathrm{O}_{3}$ and Lu $\mathrm{Lu}_{2} \mathrm{O}_{3}$ ceramics. Opt. Lett. 2011, 36, 4284-4286. [CrossRef] [PubMed]

91. Balabanov, S.S.; Gavrishchuk, E.M.; Drobotenko, V.V.; Kut'in, A.M.; Polyakov, V.S.; Storozheva, T.I. Obtaining nanodisperse powders of neodymium-activated yttrium aluminum garnet by self-propagating high-temperature synthesis method. Inorg. Mater. 2009, 45, 157-161. [CrossRef]

92. Balabanov, S.S.; Gavrishchuk, E.M.; Drobotenko, V.V.; Katkova, E.E.; Krylov, V.A.; Storozheva, T.I.; Chernova, O.Y. Preparation of weakly agglomerated yttrium aluminum garnet powders by burning a mixture of yttrium aluminum hydroxynitrates, urea, and acetic acid. Inorg. Mater. 2012, 48, 407-409. [CrossRef]

93. Balabanov, S.S.; Gavrishchuk, E.M.; Drobotenko, V.V.; Plekhovich, A.D.; Rostokina, E.E. Effect of the composition of starting yttrium aluminum hydroxide sols on the properties of yttrium aluminum garnet powders. Inorg. Mater. 2014, 50, 1030-1034. [CrossRef]

94. Balabanov, S.S.; Gavrishchuk, E.M.; Permin, D.A. Synthesis and properties of yttrium hydroxyacetate sols. Inorg. Mater. 2012, 48, 500-503. [CrossRef]

95. Balabanov, S.S.; Gavrishchuk, E.M.; Drobotenko, V.V.; Palashov, O.V.; Rostokina, E.Y.; Yavetskiy, R.P. A new approach to $\mathrm{Y}_{3} \mathrm{Al}_{5} \mathrm{O}_{12}$ transparent ceramics by vacuum sintering of spray-dried xerogels. Ceram. Int. 2016, 42, 961-965. [CrossRef]

96. Balabanov, S.S. Production of submicron $\mathrm{Al}_{2} \mathrm{O}_{3}$ powders by electrochemical dissolution of aluminum in the presence of nitric acid. Funct. Mater. 2018, 25, 788-794. [CrossRef]

97. Balabanov, S.S.; Gavrishchuk, E.M.; Rostokina, E.Y.; Plekhovich, A.D.; Kuryakov, V.N.; Amarantov, S.V.; Khamaletdinova, N.M.; Yavetskiy, R.P. Colloid chemical properties of binary sols as precursors for YAG optical ceramics. Ceram. Int. 2016, 42, 17571-17580. [CrossRef]

98. Osipov, V.V.; Platonov, V.V.; Lisenkov, V.V.; Podkin, A.V.; Zakharova, E.E. Production of nanopowders of oxides by means of fiber and pulse-periodical $\mathrm{CO}_{2}$ lasers. Phys. Status Solidi C 2013, 10, 926-932. [CrossRef]

99. Snetkov, I.L.; Palashov, O.V.; Osipov, V.V.; Mukhin, I.B.; Maksimov, R.N.; Shitov, V.A.; Luk'yashin, K.E. Continuous-wave 80-W lasing in Yb: YAG ceramics. Quantum Electron. 2018, 48, 683-685. [CrossRef]

100. Snetkov, I.L.; Palashov, O.V.; Osipov, V.V.; Mukhin, I.B.; Maksimov, R.N.; Shitov, V.A.; Luk'yashin, K.E. Investigation of lasing characteristics of domestic Yb: YAG laser ceramics. Quantum Electron. 2016, 46, 586-588. [CrossRef]

101. Luo, D.; Zhang, J.; Xu, C.; Qin, X.; Tang, D.; Ma, J. Fabrication and laser properties of transparent Yb:YAG ceramics. Opt. Mater. 2012, 34, 936-939. [CrossRef]

102. Perevezentsev, E.; Mukhin, I.; Vadimova, O.; Palashov, O.; Khazanov, E.; Luo, D.; Zhang, J.; Tang, D. Yb:YAG ceramics application for high energy cryogenic disk amplifier development. Phys. Status Solidi 2013, 210, 1232-1234. [CrossRef]

103. Permin, D.A.; Kurashkin, S.V.; Novikova, A.V.; Savikin, A.P.; Gavrishchuk, E.M.; Balabanov, S.S.; Khamaletdinova, N.M. Synthesis and luminescence properties of $\mathrm{Yb}$-doped $\mathrm{Y}_{2} \mathrm{O}_{3}, \mathrm{Sc}_{2} \mathrm{O}_{3}$ and $\mathrm{Lu}_{2} \mathrm{O}_{3}$ solid solutions nanopowders. Opt. Mater. 2018, 77, 240-245. [CrossRef]

104. Permin, D.A.; Balabanov, S.S.; Snetkov, I.L.; Palashov, O.V.; Novikova, A.V.; Klyusik, O.N.; Ladenkov, I.V. Hot pressing of $\mathrm{Yb}: \mathrm{Sc}_{2} \mathrm{O}_{3}$ laser ceramics with LiF sintering aid. Opt. Mater. 2020, 100, 109701. [CrossRef]

105. Balabanov, S.S.; Gavrishchuk, E.M.; Kut'in, A.M.; Permin, D.A. Self-propagating high-temperature synthesis of $\mathrm{Y}_{2} \mathrm{O}_{3}$ powders from $\mathrm{Y}\left(\mathrm{NO}_{3}\right)_{3 x}\left(\mathrm{CH}_{3} \mathrm{COO}\right)_{3(1-\mathrm{x})} \cdot \mathrm{nH}_{2} \mathrm{O}$. Inorg. Mater. 2011, 47, 484-488. [CrossRef]

106. Permin, D.A.; Novikova, A.V.; Gavrishchuk, E.M.; Balabanov, S.S.; Sorokin, A.A. Self-propagating high-temperature synthesis of $\mathrm{Lu}_{2} \mathrm{O}_{3}$ powders for optical ceramics. Inorg. Mater. 2017, 53, 1330-1335. [CrossRef] 
107. Chaika, M.; Balabanov, S.S.; Permin, D. Optical spectra and gain properties of $\mathrm{Er}^{3+}: \mathrm{Lu}_{2} \mathrm{O}_{3}$ ceramics for eye-safe $1.5-\mu \mathrm{m}$ lasers. Opt. Mater. 2021, 112, 110785. [CrossRef]

108. Balabanov, S.S.; Bykov, Y.V.; Egorov, S.V.; Eremeev, A.G.; Gavrishchuk, E.M.; Khazanov, E.A.; Mukhin, I.B.; Palashov, O.V.; Permin, D.A.; Zelenogorsky, V.V. Transparent $\mathrm{Yb}:(\mathrm{YLa})_{2} \mathrm{O}_{3}$ ceramics produced by self-propagating high-temperature synthesis and microwave sintering. Opt. Mater. 2013, 35, 727-730. [CrossRef]

109. Balabanov, S.S.; Bykov, Y.V.; Egorov, S.V.; Eremeev, A.G.; Gavrishchuk, E.M.; Khazanov, E.A.; Mukhin, I.B.; Palashov, O.V.; Permin, D.A.; Zelenogorskii, V. V Yb:(YLa) ${ }_{2} \mathrm{O}_{3}$ laser ceramics produced by microwave sintering. Quantum Electron. 2013, 43, 396-400. [CrossRef]

110. Snetkov, I.L.; Mukhin, I.B.; Balabanov, S.S.; Permin, D.A.; Palashov, O.V Efficient lasing in Yb:(YLa) ${ }_{2} \mathrm{O}_{3}$ ceramics. Quantum Electron. 2015, 45, 95-97. [CrossRef]

111. Ivanov, M.; Kopylov, Y.; Kravchenko, V.; Li, J.; Pan, Y.; Kynast, U.; Leznina, M.; Strek, W.; Marciniak, L.; Palashov, O.; et al. Optical, luminescent and laser properties of highly transparent ytterbium doped yttrium lanthanum oxide ceramics. Opt. Mater. 2015, 50, 15-20. [CrossRef]

112. Snetkov, I.L.; Mukhin, I.B.; Palashov, O.V. Comparative characteristics of $\mathrm{Yb}:(\mathrm{YLa})_{2} \mathrm{O}_{3}$ laser ceramics. Quantum Electron. 2016, 46 , 193-196. [CrossRef]

113. Permin, D.A.; Balabanov, S.S.; Novikova, A.V.; Snetkov, I.L.; Palashov, O.V.; Sorokin, A.A.; Ivanov, M.G. Fabrication of Yb-doped $\mathrm{Lu}_{2} \mathrm{O}_{3}-\mathrm{Y}_{2} \mathrm{O}_{3}-\mathrm{La}_{2} \mathrm{O}_{3}$ solid solutions transparent ceramics by self-propagating high-temperature synthesis and vacuum sintering. Ceram. Int. 2019, 45, 522-529. [CrossRef]

114. Maksimov, R.N.; Shitov, V.A.; Volkov, M.R.; Vadimova, O.L.; Snetkov, I.L. Spectroscopic and laser characteristics of ceramics based on $\mathrm{Yb}^{3+}$-doped $\mathrm{Lu}_{2} \mathrm{O}_{3}-\mathrm{Y}_{2} \mathrm{O}_{3}$ solid solution. Quantum Electron. 2018, 48, 695-698. [CrossRef]

115. Snetkov, I.L.; Silin, D.E.; Palashov, O.V.; Khazanov, E.A.; Yagi, H.; Yanagitani, T.; Yoneda, H.; Shirakawa, A.; Ueda, K.; Kaminskii, A.A. Study of the thermo-optical constants of $\mathrm{Yb}$ doped $\mathrm{Y}_{2} \mathrm{O}_{3}, \mathrm{Lu}_{2} \mathrm{O}_{3}$ and $\mathrm{Sc}_{2} \mathrm{O}_{3}$ ceramic materials. Opt. Express 2013, $21,21254$. [CrossRef] [PubMed]

116. Peters, R.; Kränkel, C.; Fredrich-Thornton, S.T.; Beil, K.; Petermann, K.; Huber, G.; Heckl, O.H.; Baer, C.R.E.; Saraceno, C.J.; Südmeyer, T.; et al. Thermal analysis and efficient high power continuous-wave and mode-locked thin disk laser operation of Yb-doped sesquioxides. Appl. Phys. B 2011, 102, 509-514. [CrossRef]

117. Snetkov, I.L.; Balashov, V.V. Thermo-optical properties of $\mathrm{Ho}: \mathrm{Y}_{2} \mathrm{O}_{3}$ ceramics. Opt. Mater. 2020, 100, 109617. [CrossRef]

118. Kuznetsov, I.; Mukhin, I.; Silin, D.; Palashov, O. Thermal conductivity measurements using phase-shifting interferometry. Opt. Mater. Express 2014, 4, 2204-2208. [CrossRef]

119. Peters, V. Growth and Spectroscopy of Ytterbium-Doped Sesquioxides, Dissertation; Shaker Verlag: Hamburg, Germany, 2001.

120. Perevezentsev, E.A.; Kuznetsov, I.I.; Mukhin, I.B.; Volkov, M.R.; Palashov, O.V. Multipass cryogenic $\mathrm{Yb}_{2} \mathrm{Y}_{2} \mathrm{O}_{3}$ ceramic disk amplifier. Appl. Phys. B 2019, 125, 141. [CrossRef]

121. Volkov, M.R.; Kuznetsov, I.I.; Mukhin, I.B. A New Method of Diagnostics of the Quality of Heavily Yb-Doped Laser Media. IEEE J. Quantum Electron. 2018, 54, 1700106. [CrossRef]

122. Boulon, G.; Lupei, V. Energy transfer and cooperative processes in $\mathrm{Yb}^{3+}$-doped cubic sesquioxide laser ceramics and crystals. J. Lumin. 2007, 125, 45-54. [CrossRef]

123. Snetkov, I.L.; Zhou, D.; Yakovlev, A.I.; Volkov, M.R.; Kuznetsov, I.I.; Mukhin, I.B.; Palashov, O.V.; Shi, Y.; Ueda, K.-I. Laser generation on $\mathrm{Yb}:$ LuAG ceramics produced by nanocrystalline pressure-less sintering in $\mathrm{H}_{2}$. Laser Phys. Lett. 2018, 15. [CrossRef]

124. Peng, Y.H.; Cheng, J.; Cheah, Y.Y.; Lai, K.S.; Lau, E.; Ang, S.K. High brightness continuous wave ceramic Yb:LuAG thin-disk laser. Opt. Express 2015, 23, 19618-19623. [CrossRef] 